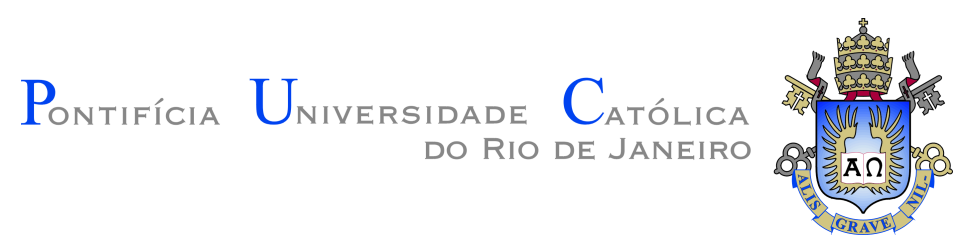

Filipe Bellio da Nóbrega

\title{
Representation of generic curves by their singularities
}

Dissertação de Mestrado

Dissertation presented to the Programa de Pós-graduação em Matemáticada PUC-Rio in partial fulfillment of the requirements for the degree of Mestre em Matemática.

Advisor: Prof. Marcos Craizer

Rio de Janeiro

July 2018 


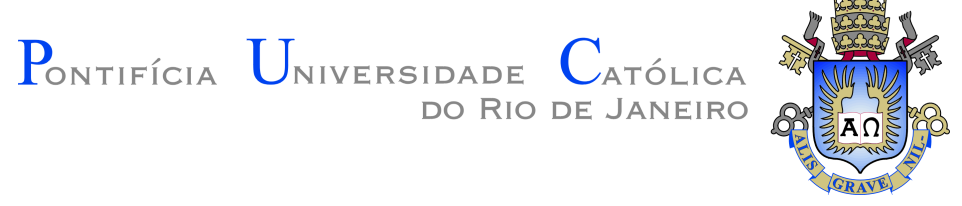

Filipe Bellio da Nóbrega

\title{
Representation of generic curves by their singularities
}

Dissertation presented to the Programa de Pós-graduação em Matemáticada PUC-Rio in partial fulfillment of the requirements for the degree of Mestre em Matemática. Approved by the undersigned Examination Committee.

\author{
Prof. Marcos Craizer \\ Advisor \\ Departamento de Matemática - PUC-Rio \\ Prof. Étienne Ghys \\ École Normale Supérieure de Lyon - ENS-Lyon \\ Prof. Paul Schweitzer \\ Departamento de Matemática - PUC-Rio \\ Prof. Nicolau Saldanha \\ Departamento de Matemática - PUC-Rio \\ Prof. Márcio da Silveira Carvalho \\ Vice Dean of Graduate Studies \\ Centro Técnico Científico - PUC-Rio
}

Rio de Janeiro, July the 17 th, 2018 
All rights reserved.

Filipe Bellio da Nóbrega

Majored in mathematics by Pontifícia Universidade Católica do Rio de Janeiro (Rio de Janeiro, Brazil)

Bibliographic data

Nóbrega, Filipe Bellio da

Representation of generic curves by their singularities / Filipe Bellio da Nóbrega; advisor: Marcos Craizer. - Rio de janeiro: PUC-Rio, Departamento de Matemática, 2018.

v., $67 \mathrm{f:}$ il. ; $30 \mathrm{~cm}$

Dissertação (mestrado) - Pontifícia Universidade Católica do Rio de Janeiro, Departamento de Matemática.

Inclui bibliografia

1. Matemática - Teses. 2. Pontos duplos. 3. Tangentes duplas. 4. Curvas localmente convexas. 5. Invariantes de Arnold. 6. Fórmula de Whitney. I. Craizer, Marcos. II. Pontifícia Universidade Católica do Rio de Janeiro. Departamento de Matemática. III. Título. 
To my family, whose loving and unconditional care gave me every support needed.

To all my friends who have shared interesting math problems and made my time at PUC-Rio so enjoyable. To Maitê Ramos and Mariana Alves, who have taught me so much while brilliantly leading PECEP alongside me. Finally, to all my students who have strived to achieve a better life through education despite all the hardship. You inspire me. 


\section{Acknowledgments}

I would like to deeply thank my advisor, Marcos Craizer, who has been a true mentor to me. It's been five years of continued study, I owe a great portion of my mathematical upbringing to you. It has been a pleasure to work together.

Then I wish to thank Étienne Ghys, Paul Schweitzer and Nicolau Saldanha for the prompt acceptance to be part of the jury. You are all professors and mathematicians of my highest esteem, it is an honor to have you present.

Finally, I wish to thank the Mathematics Department at PUC-Rio and FAPERJ for the financial support during my master's degree. 


\section{Abstract}

Nóbrega, Filipe Bellio da; Craizer, Marcos (Advisor). Representation of generic curves by their singularities. Rio de Janeiro, 2018. 67p. Dissertação de Mestrado - Departamento de Matemática, Pontifícia Universidade Católica do Rio de Janeiro.

The aim of this work is to study the topological and geometric properties of closed generic immersed curves in the plane. In this case, generic means that the curve can only have double points without a common tangent. One can label the singularities using $n$ symbols, such as a1, .., an. Going around the curve, a cyclic word of length $2 \mathrm{n}$ is produced. However, not every word is related to a planar curve, there are requirements on its combinatorics, the first of which was found by Gauss. Advances were made in the study of locally convex curves on the plane, the sphere and the projective plane.

\section{Keywords}

Double points Double tangents Locally convex curves Arnold's invariant Whitney's formula 


\section{Resumo}

Nóbrega, Filipe Bellio da; Craizer, Marcos. Representação de curvas genéricas por suas singularidades. Rio de Janeiro, 2018. 67p. Dissertação de Mestrado - Departamento de Matemática, Pontifícia Universidade Católica do Rio de Janeiro.

O objetivo desta pesquisa é estudar as propriedades geométricas e topológicas de curvas genéricas imersas no plano. Neste caso ser genérica significa que a curva só pode ter pontos duplos sem tangentes comuns nas duas passagens. Pode-se nomear as n singularidades da curva usando símbolos como a1, ..., an. Percorrendo a curva, produz-se uma palavra cíclica de tamanho 2n. Entretanto, nem toda palavra está relacionada a uma curva plana, há requisitos sobre a sua combinatória, o primeiro dos quais foi descoberto por Gauss. Avanços foram realizados no estudo de curvas localmente convexas no plano, na esfera e no plano projetivo.

\section{Palavras-chave}

Pontos duplos Tangentes duplas Curvas localmente convexas Invariantes de Arnold Fórmula de Whitney 


\section{Table of contents}

1 Introduction $\quad 9$

2 Cyclic Words of Planar Curves $\quad 11$

2.1 Closed curves, cyclic words and chord diagrams 11

$\begin{array}{ll}2.2 & \text { The signed Gauss' problem } \\ \end{array}$

3 Classes of Curves on the Sphere $\quad 18$

$\begin{array}{lll}3.1 & \text { Defining classes of curves } & 18\end{array}$

3.2 Study of cycles 25

$4 \quad$ The Words of Locally Convex Curves $\quad 31$

4.1 Necessary condition on the combinatorics 31

5 Arnold and Whitney's Contribution $\quad 36$

$\begin{array}{lll}5.1 & \text { Arnold's generic curve invariants } & 36\end{array}$

5.2 Whitney's formula for double points 43

$6 \quad$ Bjerre and Double Tangents 48

6.1 Relating double points, double tangents and inflectional points 48

6.2 The special family $\mathfrak{B} \quad 52$

6.3 Possible routes for generalisation $\quad 56$

$\begin{array}{lll}7 & \text { Closed curves on the projective plane } & \mathbf{5 8}\end{array}$

$\begin{array}{lll}7.1 & \text { Relocating to the projective plane } & 58\end{array}$

7.2 The word of double tangents 61

$\begin{array}{lll}7.3 & \text { The dual curve } & 62\end{array}$

$\begin{array}{lc}\text { Bibliography } & 66\end{array}$ 


\section{1 \\ Introduction}

The study that originated this work began in January 2017 when Professor Étienne Ghys gave a summer course at IMPA named "Tópicos da Teoria das Singularidades: Combinatória, Topologia e Álgebra”. At a given point some topological questions about closed curves on the plane were presented and it sparked curiosity and interest on the subject.

The main focus of the research is the relation between the combinatorics of cyclic words and geometric and topological properties of closed generic curves. In this way, established results in those areas of Mathematics were considered and applied to the study of the singularities of planar curves.

The main achievement of the work is the approach dedicated to locally convex curves that led to the discovery of a necessary condition on the combinatorics of the cyclic words of their double points. Furthermore, this study was not restricted just to the Euclidean plane $\mathbb{R}^{2}$; curves were also considered on the sphere $\mathbb{S}^{2}$ and on the real projective plane $\mathbb{R P}^{2}$.

In the second chapter, the original problem posed by Gauss is explained and discussed. A solution presented by Ghys in his most recent book is displayed and proved.

The third and fourth chapters are dedicated to the analysis of locally convex curves on the plane. First we define some classes of curves on the sphere that bring together curves that have the same cyclic word of double points. Then, we define cycles and use them to state the newfound necessary condition.

The fifth chapter is dedicated to the work of two authors who made major contributions on the subject, namely Arnold and Whitney. We present Arnold's theory of invariants for generic immersions of closed curves and discuss Whitney's formula that relates the double points to the index of the curve. A new proof for this theorem is also provided.

Another author is highlighted in the sixth chapter, Fabricius-Bjerre. His work concerns the double tangents of the closed curves. We present some formulas that he has derived and point out a special family of curves with particular properties.

The final chapter is reserved for closed curves on the projective plane. We 
give some examples in order to better understand their fundamental properties. The theory developed thus far is applied to this new setting and the concept of duality is explored. Some questions remain open, but hopefully this work will encourage more people to study this fascinating subject.

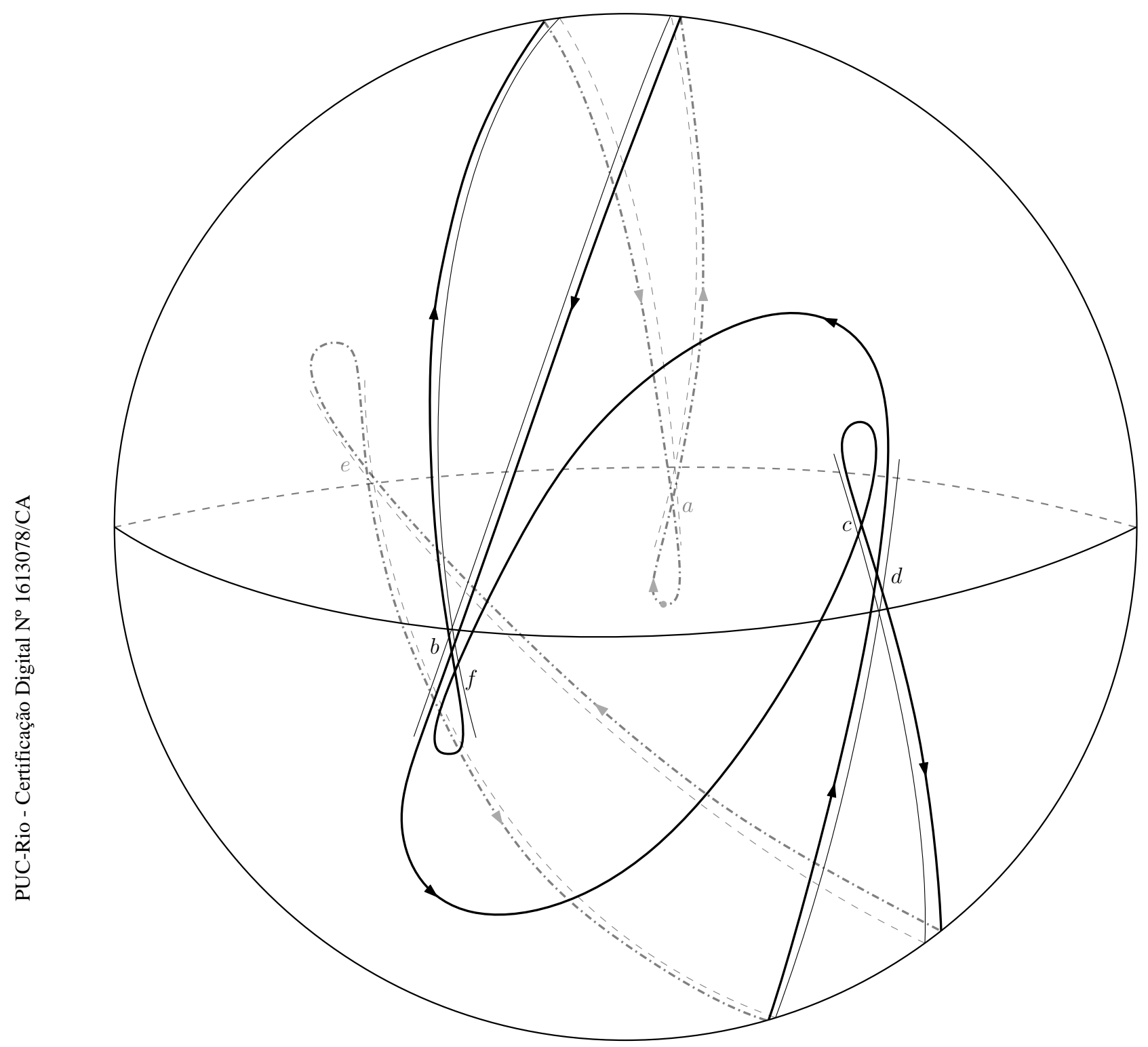




\section{2}

\section{Cyclic Words of Planar Curves}

\section{1}

\section{Closed curves, cyclic words and chord diagrams}

This work concerns the planar curves and their topological properties, which is a subject that has been studied for hundreds of years. Gauss himself wrote some notes about it, and this will be our starting point. Consider a closed generic oriented immersed curve $i: \mathbb{S}^{1} \rightarrow \mathbb{R}^{2}$ in the plane. In this case, generic means that the curve can only have double points without a common tangent, i.e., if the curve crosses itself at a point, there can only be two arcs passing through that point and they cannot have the same tangent there.

Such a curve has only finitely many double points, so we can label them using $n$ symbols, such as $a_{1}, \ldots, a_{n}$. If one goes around the curve, each double point is passed by twice, and we get a cyclic word of length $2 n$, where each of these $a_{i}$ 's appears two times. Therefore, it is possible to construct a chord diagram which represents the topological behaviour of the curve's selfintersections.
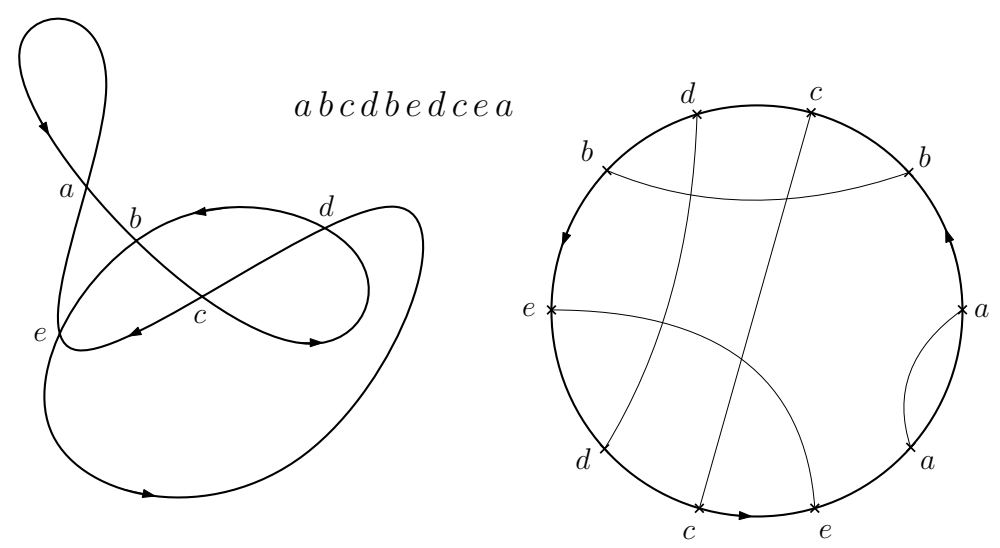

The problem that interested Gauss was how to determine whether a chord diagram is related to a planar curve or not. So given a cyclic word with $n$ pairs of symbols, is there a planar curve whose double points generate it? Gauss found out a first necessary condition. 
Proposition 2.1 If a cyclic word with $n$ pairs of symbols is associated to a planar curve, then given any $a_{i}$ there must be an even number of letters between each of its occurrences.

To see why this is true, first we need a lemma.

Lemma 2.2 Any two closed planar curves that intersect transversally have an even number of intersection points.

Proof of the Lemma.

Take one of the curves and slide it through a generic path of translations until the curves are separated. We just need to examine how intersection points appear or disappear under such generic processes. It is easy to see that they do so in pairs. Therefore, as at the end there are zero intersection points, there must be an even number of them at the beginning.

Proof of the Proposition.

Now, consider a closed generic oriented immersed curve $\gamma$ and a double point $a_{i}$, like the following.

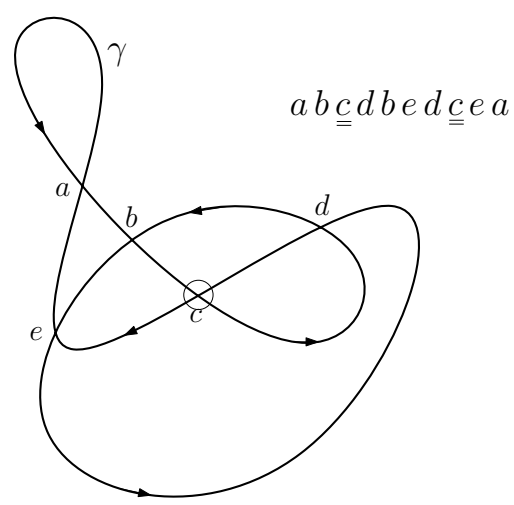

Its chord on the chord diagram divides the circle into two intervals $I_{1}$ and $I_{2}$, each of which represents a closed curve, $\gamma_{1}$ and $\gamma_{2}$, that begin and end at $a_{i}$. Moving them slightly so they become transversal and do not cross near $a_{i}$, we have two closed planar curves.

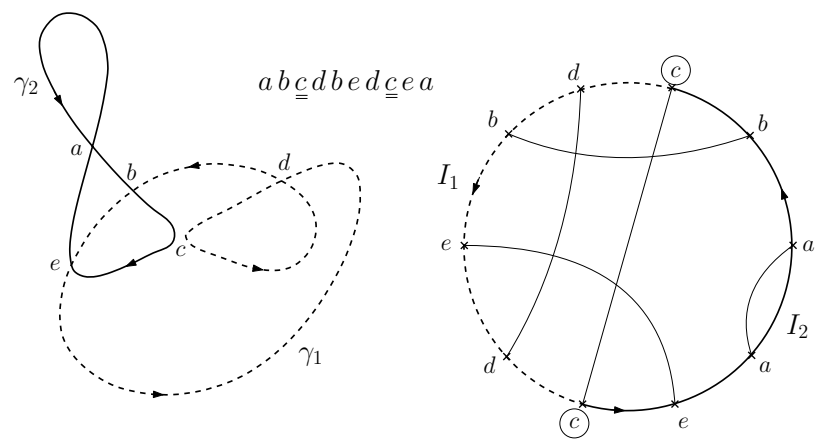


By the lemma, they must have an even number of intersection points. Looking back at the chord diagram, we see that the letters in the interval $I_{1}$ either correspond to double points of the curve $\gamma_{1}$, in which case the letter appears twice in $I_{1}$, or to intersection points between $\gamma_{1}$ and $\gamma_{2}$, in which case it appear only once in each interval. Therefore, the number of letters in each interval must be even.

However, as Gauss knew very well, this condition alone is not enough. There have been many different ways to solve the problem, mainly topological and combinatorial ones. The following is an interesting solution presented by Étienne Ghys in his most recent book (1). We will follow his reasoning closely.

\section{2}

\section{The signed Gauss' problem}

We will consider an easier version of the problem, the signed problem of Gauss. If the plane is oriented, it is possible to define an order to the two tangent vectors of each double point, so that one is "the first" and the other "the second". Going around the curve, if we cross a double point $a_{i}$ along "the first" branch we will denote it as $a_{i}^{+}$, and we will use $a_{i}^{-}$otherwise. In that way, we obtain signed words and we can think, equivalently, of oriented chord diagrams, where each chord goes from the positive + end to the negative one. So the signed Gauss's problem is to determine whether a signed cyclic word comes from some generic curve in the plane.

To solve this problem we will construct a topological surface in a clever way. For each letter $a_{1}, \ldots, a_{n}$ we will construct a cross with a positive and a negative oriented arm.

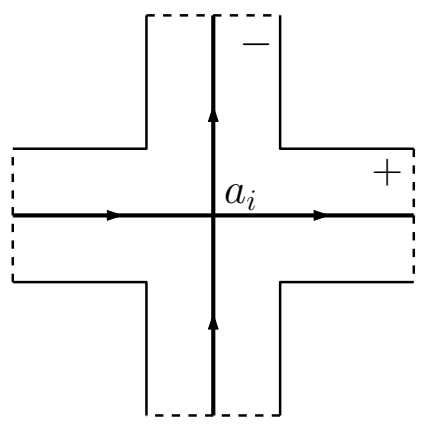

A signed cyclic word determines uniquely how to glue those crosses. The result of this process is an oriented surface $S$ with boundary containing an immersed oriented curve $\gamma$ which originates our original signed cyclic word. So if $S$ is planar, i.e., if it is possible to embed it in the plane, the problem is solved, since we constructed a curve in the plane. Conversely, if the word 
comes from a curve in the plane, it is clear that some neighbourhood of its image is a union of crosses assembled as in $S$.

Therefore, a signed cyclic word $w$ is associated to an immersed planar curve if and only if $S$ is planar.

How does one find out if $S$ is planar? Let $k$ be the number of boundary components os $S$. Now glue a disc to each of its boundary components to obtain a new surface $\hat{S}$. Because the surface $S$ has the homotopy type of a graph with $n$ vertices and $2 n$ edges, the Euler-Poincaré characteristic of $\hat{S}$ is $n-2 n+k$. It is well known that compact oriented surfaces without boundary are classified by their Euler-Poincaré characteristic, and as corollary, $S$ is planar if and only if $k-n=2$.

So there is an algorithm to decide whether $w$ is realizable: just glue the crosses and count the number of components of the border. There is a planar curve with that signed word if and only if such number is $n+2$. However, this algorithm doesn't give us a complete understanding of the matter, and it can be quite hard to manage the surface $S$.

Fortunately, there is another way to solve the problem. It follows from the classification of compact oriented surfaces with boundary that such a surface is planar if and only if any two closed transverse curves intersect in an even number of points. If the genus of the surface is greater than or equal to 1 , it contains a punctured torus which contains two curves that intersect at a single point. Hence, to verify if the genus of $S$ is 0 , it suffices to find a basis of its homology $H_{1}(S ; \mathbb{Z} / 2 \mathbb{Z})$ modulo 2 , and to compute the intersection.

We start to consider some closed curves on $S$. First, there is $\gamma$, the original curve that goes over every cross. For every $i=1,2, \ldots, n$, we consider the interval from $a_{i}^{+}$to $a_{i}^{-}$, which defines a loop $\gamma_{i}$. It begins at the center of $a_{i}$ 's cross, leaving it through the positive arm. Then it goes straight over the other crosses, that is, it never turns to change its direction. When it finally comes back to its original cross through the negative arm, it connects at the center, giving the closed curve $\gamma_{i}$. In that way the intersection of $\gamma_{i}$ with a cross different from the one labled $a_{i}$ is either empty, or a straight segment, or two perpendicular segments.

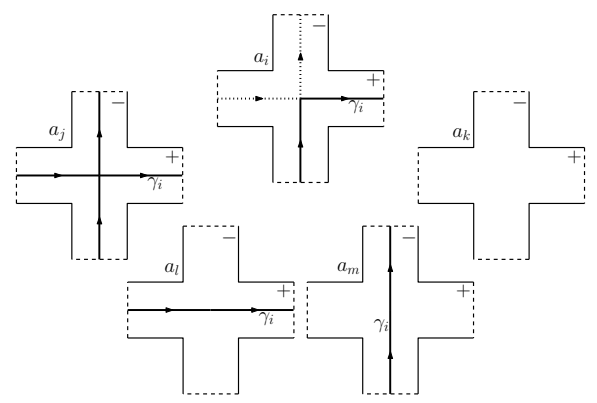


Lemma 2.3 The classes $[\gamma],\left[\gamma_{i}\right]_{1 \leq i \leq n}$ define a basis of $H_{1}(S ; \mathbb{Z} / 2 \mathbb{Z})$.

Proof. The surface $S$ has the homotopy type of a connected graph with $n$ vertices and $2 n$ edges, thus its Euler-Poincaré characteristic is $-n$. Since this invariant is related to the Betti numbers $b_{i}$, which are the the rank of the $i$-th homology group, through the following formula:

$$
\chi=b_{0}-b_{1}+b_{2}-b_{3}+\ldots
$$

and $b_{j}=0, j \geq 2$, we can conclude that $-n$ is equal to 1 minus the rank of $H_{1}(S ; \mathbb{Z} / 2 \mathbb{Z})$. Therefore this rank is $(n+1)$.

In order to prove the lemma we just have to show that the elements $[\gamma],\left[\gamma_{i}\right]_{1 \leq i \leq n}$ are linearly independent in $H_{1}(S ; \mathbb{Z} / 2 \mathbb{Z})$.

Any $\operatorname{arc} \sigma$ in $S$ with endpoints in the boundary of $S$ defines a linear form in $H_{1}(S ; \mathbb{Z} / 2 \mathbb{Z})$ : just count intersection points with $\sigma$ modulo 2. Consider an arc such as the following on an arbitrary cross. One can see that $\gamma$ intersects it in only one point, so $[\gamma]$ is not trivial.

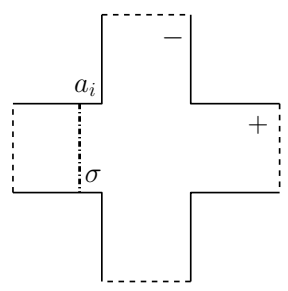

For $\left[\gamma_{i}\right]$, consider $\sigma_{i}$ as the sum of the two curves in the cross associated to the letter $a_{i}$, as in the image below.

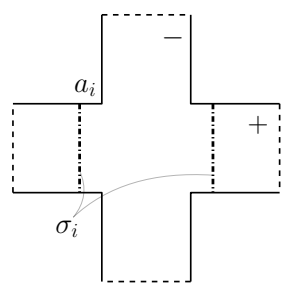

The intersection of $\gamma_{j}$ with $\sigma_{i}$ is 0 if $i \neq j$ and 1 if $i=j$. In addition, the intersection of $\gamma$ with $\sigma_{i}$ is always 0 . Therefore one can use these $n+1$ linear forms $\sigma_{i}$ and $\sigma$ to show that $[\gamma],\left[\gamma_{i}\right]_{1 \leq i \leq n}$ are linearly independent and the lemma is proved.

Now, the surface $S$ has genus zero if and only if the intersection number of the loops $\gamma, \gamma_{i}$ are all 0 modulo 2 .

The self intersection number of any closed curve on any orientable surface is 0 (over $\mathbb{Z}$ and hence over $\mathbb{Z} / 2 \mathbb{Z}$ ). 
Let us compute the intersection number of $\gamma$ and $\gamma_{i}$, denoted by $\gamma \cdot \gamma_{i}$. In order to make them transversal, move $\gamma$ slightly to its right, originating $\gamma^{\prime}$. It follows that the intersection number of $\gamma$ and $\gamma_{i}$ is the number of letters between $a_{i}^{+}$and $a_{i}^{-}$. In this way, we recovered Gauss's necessary condition. We will assume from now on that it is satisfied.

Next, we will compute the intersection number modulo 2 of $\gamma_{i}$ and $\gamma_{j}$, namely $\gamma_{i} \cdot \gamma_{j}$. We find two different situations.

If the letters $a_{i}^{ \pm}, a_{j}^{ \pm}$are not linked, there are two disjoint intervals I, J in the circle (or in the cyclic word) whose endpoints are $a_{i}^{+}, a_{i}^{-}$and $a_{j}^{+}, a_{j}^{-}$ respectively. Since $\gamma_{i}$ can be replaced by $\gamma_{i}-\gamma$ in the computation of the intersection number, it follows that $\gamma_{i} \cdot \gamma_{j}$ is the number of letters in the word with one occurrence in I and the second in $\mathrm{J}$ in this unlinked case. This is therefore a second parity condition, necessary for the realizability of $w$.

If the letters $a_{i}^{ \pm}, a_{j}^{ \pm}$are linked, the loops $\gamma_{i}$ and $\gamma_{j}$ are not transversal; they coincide on some non-trivial interval. Move $\gamma_{i}$ slightly to the right to get some $\gamma_{i}^{\prime}$, and move $\gamma_{j}$ to the left to produce $\gamma_{j}^{\prime}$. These new curves are parallel on this common part.

To count the intersections, first we take a close look at the crosses associated to $a_{i}$ and $a_{j}$.
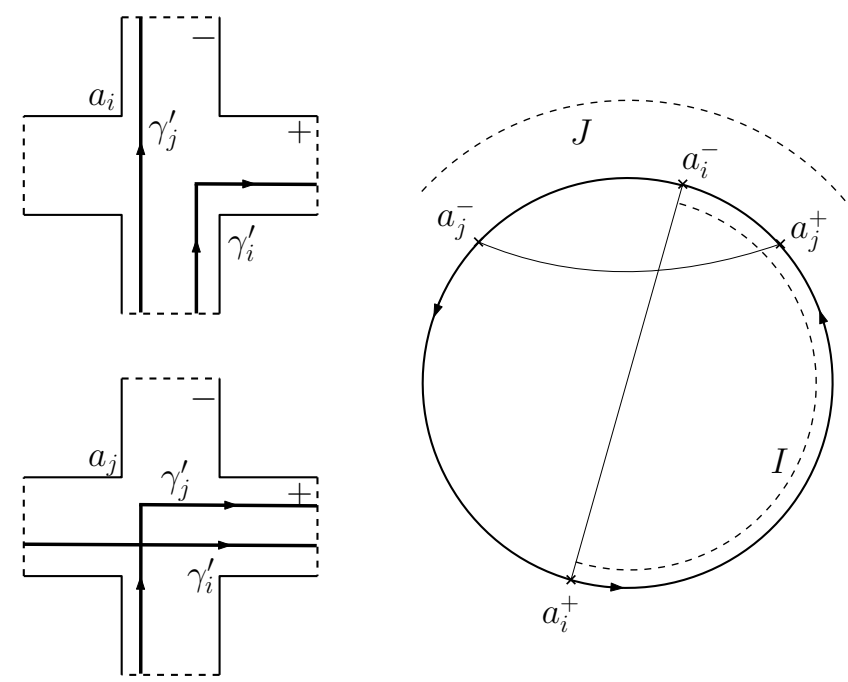

One can see that there are no intersections in the cross $a_{i}$, and there is a single one in the cross $a_{j}$. The other intersections correspond to letters between $a_{i}^{+}$and $a_{i}^{-}$whose second occurence is between $a_{j}^{+}$and $a_{j}^{-}$, except if both are in $I \cap J$, since in this case there are two intersection in the same cross, so it is irrelevant modulo 2. Hence, when $a_{i}^{ \pm}, a_{j}^{ \pm}$are linked, the intersection number $\gamma_{i} \cdot \gamma_{j}$ is equal to 1 plus the number of letters with one ocurrence in I and the other in $J \backslash I$ or with one ocurrence in $J$ and the other in $I \backslash J$.

We therefore get a very simple answer to the signed Gauss's problem. 
Theorem 2.4 A signed Gauss word is realizable by a planar immersed curve if and only if the following conditions are satisfied:

1. For every letter $a_{i}$, there is an even number of letters between $a_{i}^{+}$and $a_{i}^{-}$ (Gauss's parity condition).

2. For every $i, j$ such that the letters $a_{i}^{ \pm}$and $a_{j}^{ \pm}$are not linked, let $I, J$ be the disjoint intervals whose endpoints are $a_{i}^{+}, a_{i}^{-}$and $a_{j}^{+}, a_{j}^{-}$(excluding these points). The number of letters in the word with one occurrence in $I$ and the other in $J$ is even.

3. For every $i, j$ such that the letters $a_{i}^{ \pm}$and $a_{j}^{ \pm}$are linked, the number of letters between $a_{i}^{+}$and $a_{i}^{-}$whose other occurence is between $a_{j}^{+}$and $a_{j}^{-}$, and that do not appear twice in $I \cap J$, is odd. 


\section{Classes of Curves on the Sphere}

\section{1}

\section{Defining classes of curves}

In the previous chapter, the conditions that a signed cyclic word $w$ must meet in order to correspond to an immersed closed planar curve were shown. In this chapter we will develop the necessary tools to investigate a particular family of planar curves, namely the locally convex curves.

Definition 3.1 An immersed planar curve is said to be locally convex if it does not have any inflection point.

Since these curves have no inflection points, their concavity is always well-defined and never changes sign. Equivalently, one can say that the curve always "turns to the same side"; if you imagine yourself driving a car along the curve, you should keep steering to the same side all the way.

The main goal is to find conditions on the words of locally convex curves. First of all, there are curves which are not locally convex that can easily become so by slightly moving it, and the letters in its word cannot detect such movement.

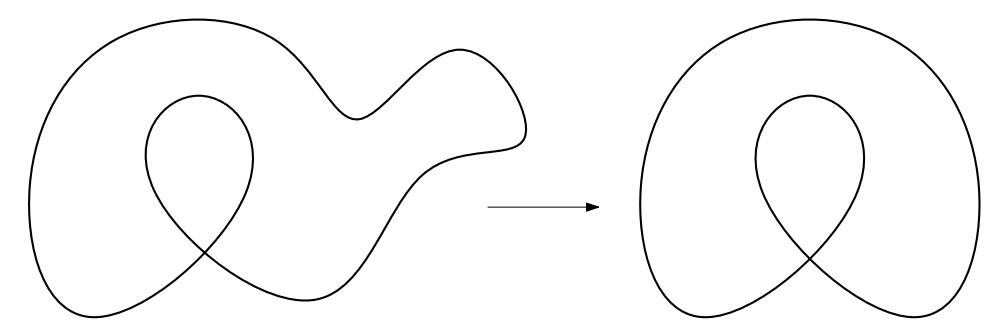

Therefore, we must consider classes of curves under continuous deformations for the reasoning to make sense. Since we want all the curves from a given class to have the same signed cyclic word, creating or getting rid of intersection points is clearly not allowed. In fact, given two closed generic oriented immersed curves $\gamma_{1}$ and $\gamma_{2}$, if one considers a generic connecting path of immersions between them, there are only three types of singular events that can occur. These are triple crossings of the immersion and self-tangencies of two types, direct self-tangencies if the tangent vectors of both branches coincide, 
and inverse self-tangencies otherwise. This fact was shown by Arnold (2) and we will examine it thoroughly in the next chapter.

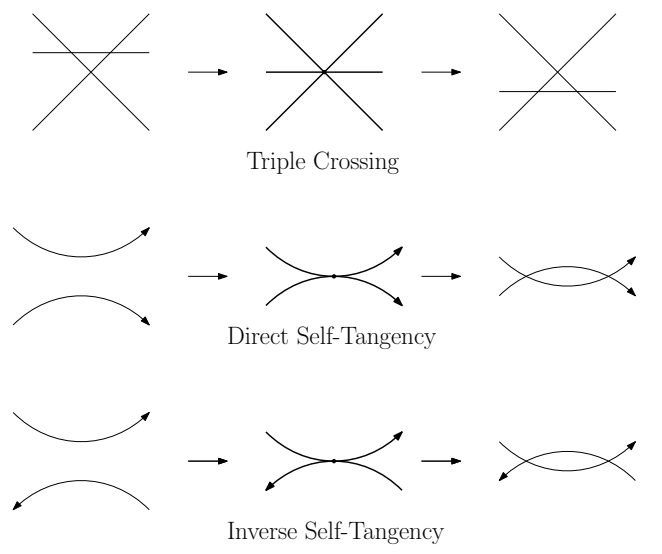

Considering two curves as being from the same class if there is a connecting path of immersions that avoids all singular events guarantees that the class has a well-defined cyclic word associated with it, because every curve in it shares the same word. However, there can be two distinct classes with the same cyclic word as it is defined so far, unfortunately. To see this, consider the trivial example of the word $a^{+} a^{-}$and look at the following two curves.
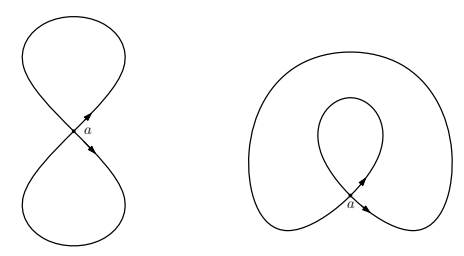

There is no generic path in the plane between these curves without a singular crossing, they nevertheless have the same word. Thus, we seek a new way to define the classes such that those curves are considered as the same. The small tweak we need is to allow the arcs of the curve to pass through infinity. Imagine the bottom arc of the figure eight curve expanding until it "reaches infinity", passes through it and appears on the other side. In this way, we connect the original curves without singular crossing and, therefore, we group them in a single class.

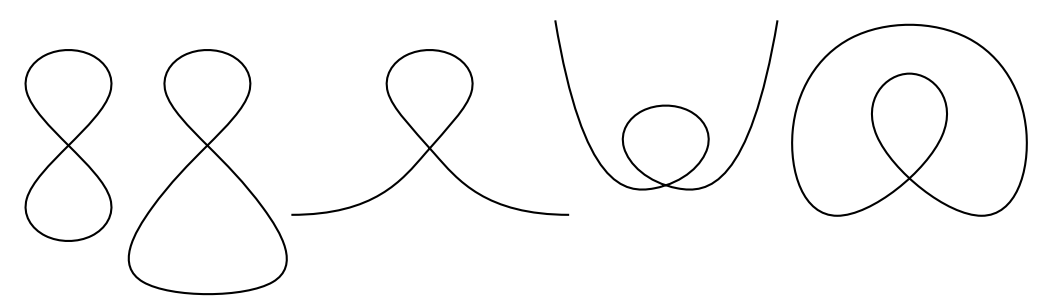


To formally define the equivalence relation over the curves, we must consider curves on the sphere rather than on the plane. The geometric interpretation of "passing through infinity" comes from the gnomonic projection.

Set a point on the sphere as its north pole and consider the tangent plane at that point. The gnomonic projection maps any point $p$ on the northern hemisphere to a point $p^{\prime}$ on the plane by taking the line through the center of the sphere and $p$. Its intersection with the plane defines the image $p^{\prime}$.

This map sends a hemisphere into the plane and it has the useful property of mapping arcs of great circles to straight lines, so the geodesics of the sphere are mapped to the geodesics of the plane. This implies that locally convex curves on the hemisphere are mapped to locally convex curves on the plane. We will use the gnomonic projection and its inverse function to work with the curves either on the sphere or the plane. Fortunately, since the sphere is orientable we can keep the sign as we defined it for the double points.

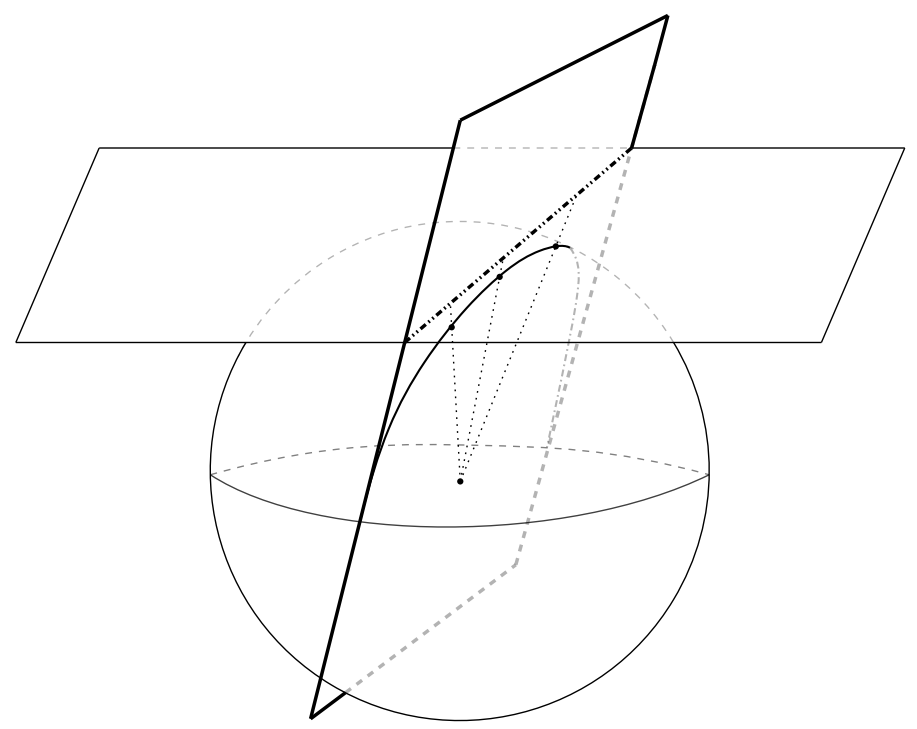

Definition 3.2 Two closed generic oriented immersed curves on the sphere are in the same equivalence class if and only if there is a connecting path of immersions that avoids all singular events.

At this point we have a satisfactory definition for the equivalence relation, for we have the following result.

Proposition 3.3 Every equivalence class of curves on the sphere has a unique signed cyclic word related to it and no word is related to more than one class.

Proof. Since there is a connecting path of immersions that preserves the signed cyclic word between any two curves in the same class, it is clear that all curves in the class have the same word. 
To show that no word is related to more than one class, consider an arbitrary signed cyclic word $w$. We shall draw a curve on the sphere according to the word's instructions and we will notice that there is no choice in the process, thus we always end on the same class when we finish. Choose any letter of $w$ to be the "first" one. We start at an arbitrary point on the sphere and draw an arc until we get to the first double point, corresponding to that first letter of the word. It is important to notice its sign, so we draw a small transversal arc appropriately oriented. Keep doing so for the new double points, until the next letter is a repeat.

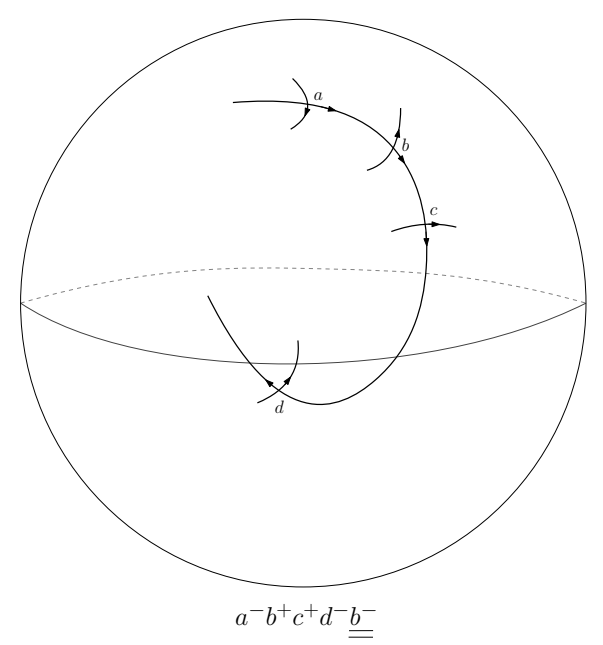

At this point we have to connect the curve to the small transversal arc already drawn crossing that double point. If we were on the plane, there would be two ways to do so, each one going around the curve drawn so far in a different way. However, on the sphere these arcs are equivalent. Since the set that cannot be crossed is connected and the extremities of the arc are in the same path-component, it is possible to pass it through the back of the sphere; so we can choose either.

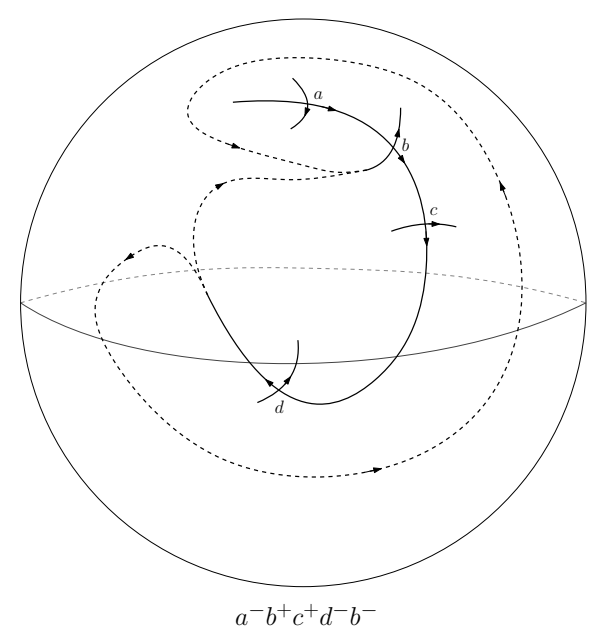


Now we have divided the sphere into two connected componentes. Since we are keeping track of the double points, we know exactly at which points the curve will cross the boundary between them.

We continue our construction, possibly adding new double points. When we come across another repeat, we might have two equivalent options as before or a previous arc might block us, leaving only one way to proceed. Either way, any curve we draw is equivalent.

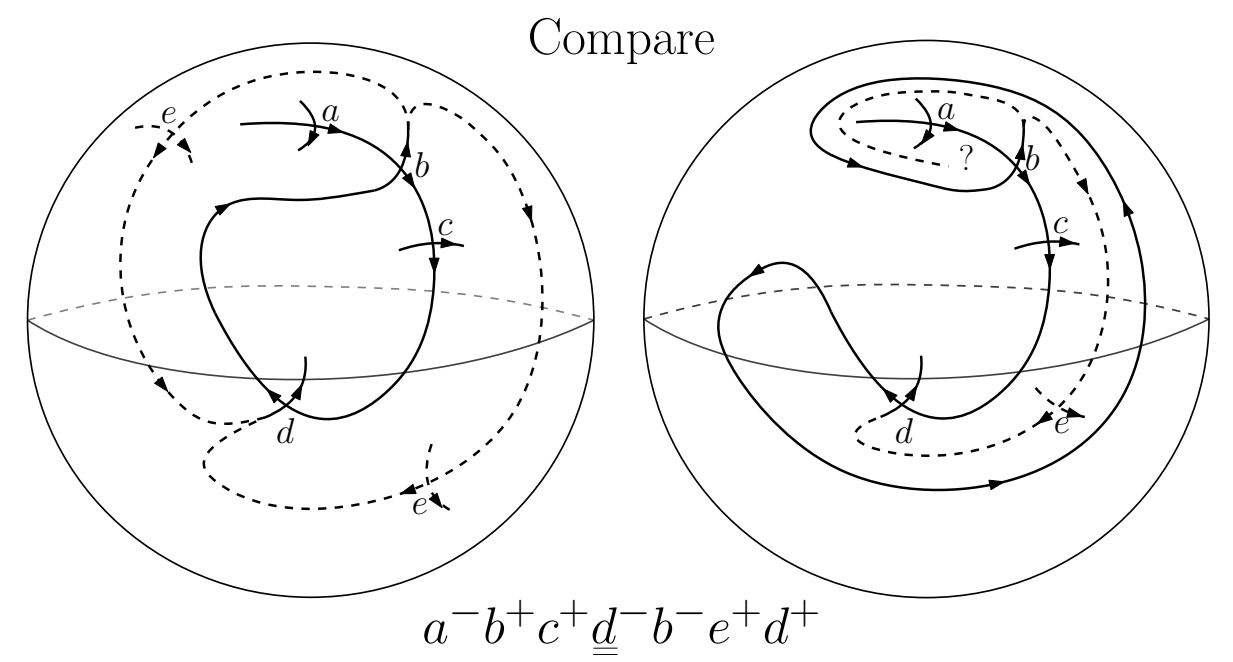

Provided that the word $w$ is realizable, i.e., it follows the conditions presented in Chapter 1, there won't be any problem that prevents us from completing our drawing, and so we get a closed curve on the sphere. As we saw, any curve we construct in that way is equivalent, therefore every realizable word is related to a single class of equivalent curves on the sphere.

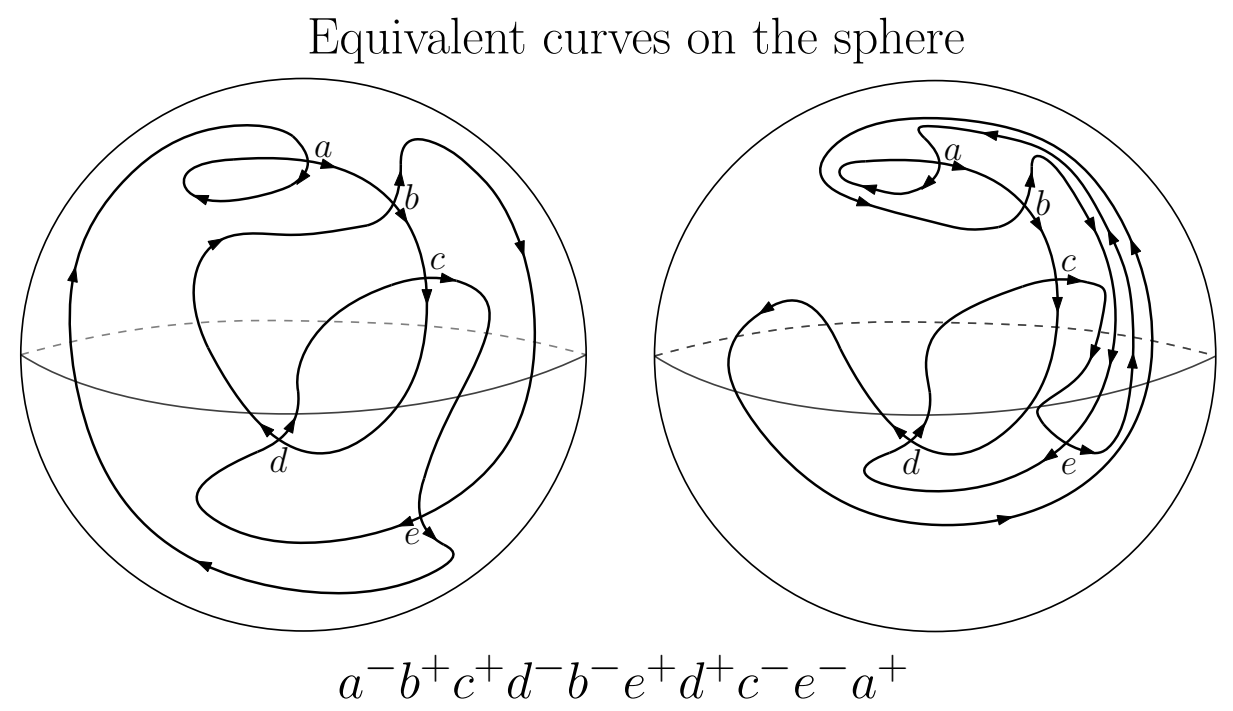

An interesting reward of the construction in the proof is that by means of the equivalent choices we get all possible different appearances of the 
curve back on the plane. Actually, the closed curve divides the sphere into a finite number of connected components as a consequence of the Jordan Curve Theorem, and by picking a point in the interior of one of them as the "south pole" and pulling up the whole curve to the northern hemisphere, the domain of the gnomonic projection, and applying it we get a planar view of a representative of the equivalence class. This process provides every distinct planar view of the class.

\section{Example: $a^{-} a^{+} b^{-} b^{+}$}
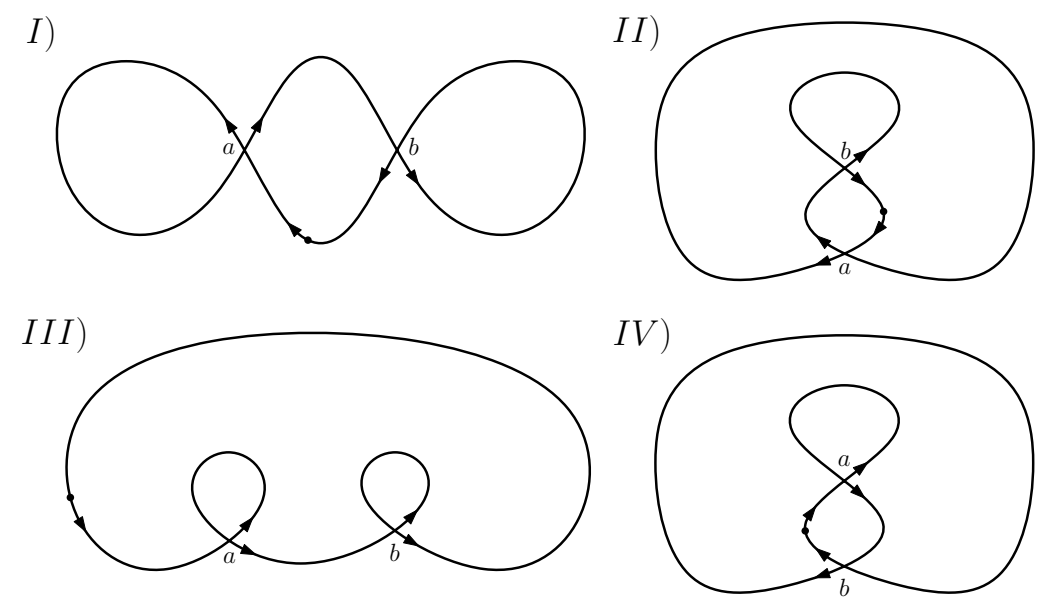

In fact, there are many ways to show that a generic immersed closed planar curve with $n$ double points divides the plane into exactly $n+2$ connected components. The curve on the plane can be seen as a planar graph, so Euler's formula applies. It has $n$ vertices, all of which have degree 4 , so the number of edges is $2 n$ by a double counting argument. Thus we have:

$$
\begin{gathered}
v-e+f=2 \\
f=n+2
\end{gathered}
$$

Another way to prove this is by taking the double points away one by one. Consider the neighbourhood of a double point, it is divided into four regions. Delete the double point and then reconnect the branches without crossings in a way that keeps the curve connected.

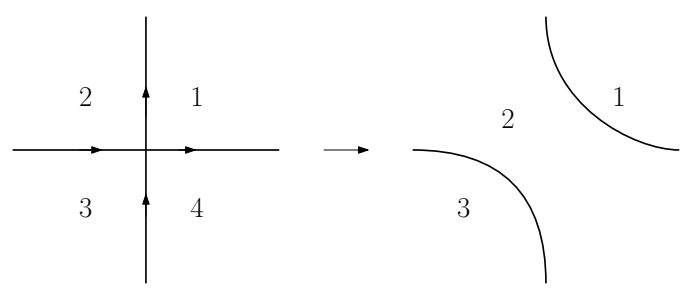


In the figure, we know that the righthand side of the curve eventually comes back from the bottom since the curve was oriented. So the only way to maintain it connected is to violate its original orientation and proceed as shown in the figure. By doing so, the regions numbered 2 and 4 come together, thus if they were not one and the same to begin with, the number of regions has decreased by 1 .

One can prove by contradiction that those regions were two distinct ones. Suppose 2 and 4 were part of the same region, so there is a path $\mu$ that does not cross the original curve going from 2 to 4 . After the maneuver we did around the double point, it is possible to close such a path $\mu$ and the Jordan Curve Theorem states that the two portions we can see of the original curve are in different connected components. Since the curve is still connected, we find that there must be a intersection between the curve and $\mu$, which is a contradiction.

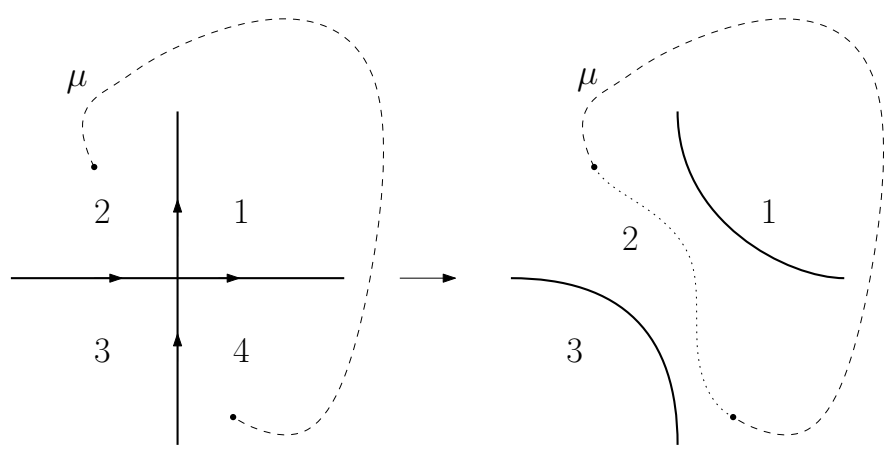

To conclude the proof, we just need to give any orientation to the new curve we have and repeat the process. After taking all $n$ double points away, we end with a closed curve with no singularities, which is topologically the same as a circle. Since it divides the plane into 2 connected components, we conclude that the original curve divides the plane into $n+2$ regions.
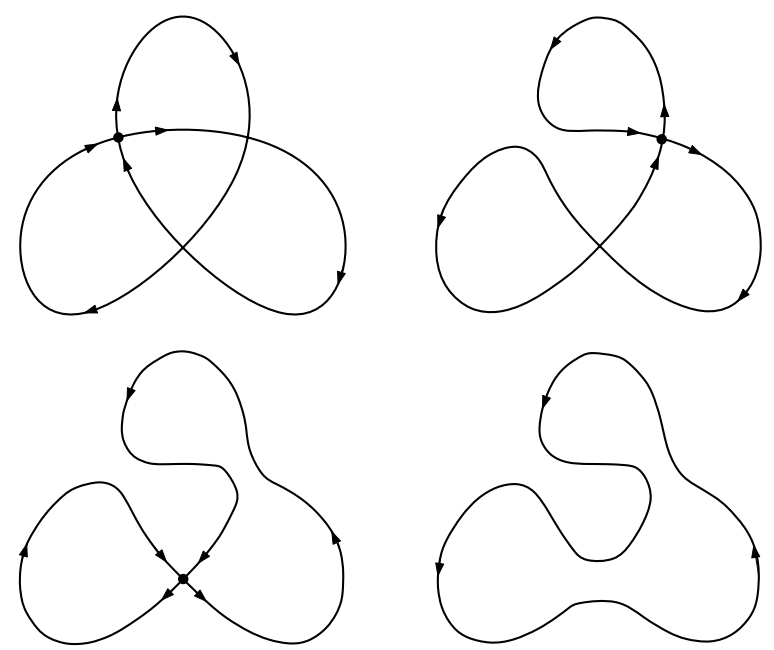
In the fifth chapter there is a third proof of this fact using Arnold's theory.

\section{2}

\section{Study of cycles}

Now that we have the desired equivalence relation, we search for requirements for there to be a locally convex curve among the representatives of the class.

One such condition was found; it concerns the orientation of cycles defined by the curve.

Definition 3.4 A cycle is a connected component of the complement of an oriented closed curve on the sphere such that its boundary's orientation never flips. Equivalently, it is possible to go around its boundary, turning corners at the double points, and never be against the original curve's orientation. The length of a cycle is the number of arcs that compose its boundary.

Fortunately, the cycles defined by the curve can be discovered just by looking at its signed cyclic word. For a pair of consecutive letters in the cyclic word to represent an arc in the boundary of a cycle, they must have opposite signs. This can be easily seen in the following picture.
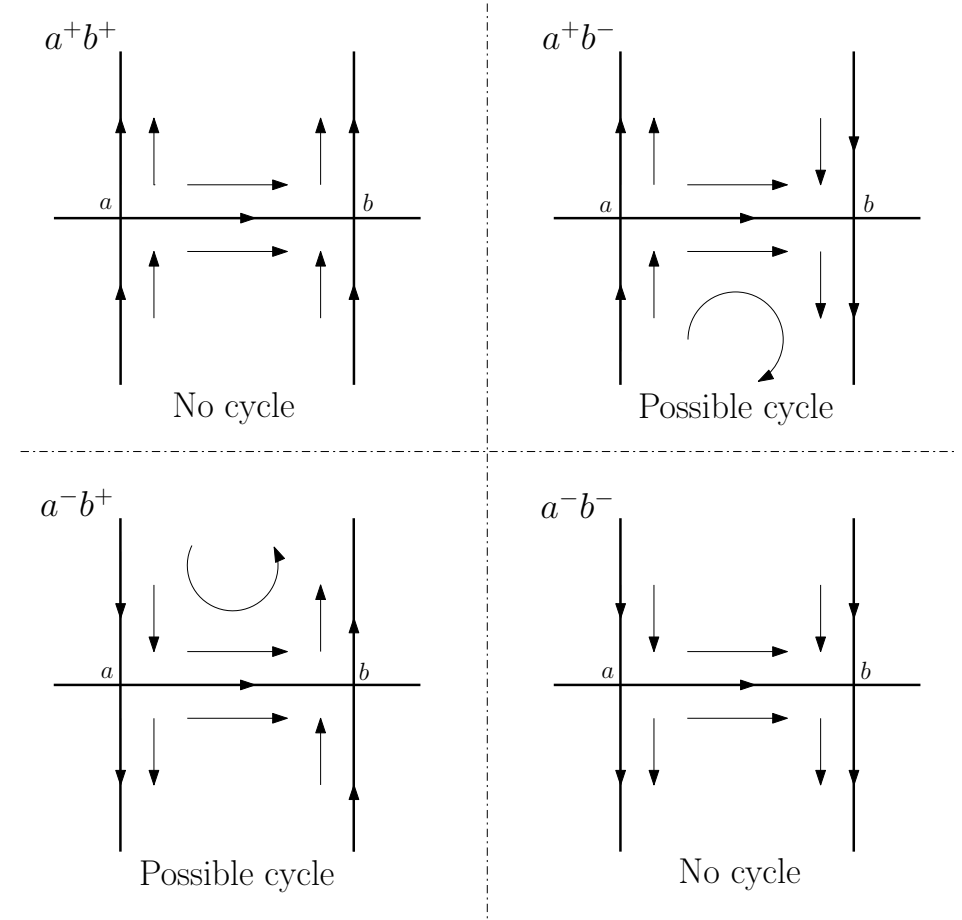

One can identify a cycle of length $n$ in the word as a sequence of $n$ pairs of letters with opposing signs, which represent the $n$ oriented arcs in the cycle's 
boundary. The simplest example is a cycle of length 1 such as $a^{+} a^{-}$. Also, every pair must have the same order of signs, either $(+,-)$ or $(-,+)$. To see this, take a look back at the previous figure, if we begin with $a^{+} b^{-}$there must be an arc that begins with $b^{+}$, so it could be $b^{+} c^{-}$, for example. This process goes on until we get back to $a^{-}$, where the cycle closes itself. The reasoning is the same for the other case. The order of the signs also determines the sense of rotation of the cycle; this can be seen in the picture above.

There are three important remarks. If the cycle has length greater than 1 , its pairs do not have to come one right after the other; they can be spread out throughout the word. In addition, they do not have to appear in any particular order; they build the cycle in steps. Finally, the same letter can be part of two different cycles, so we must look closely not to miss any. Here we have some examples to see these behaviours.

$$
a^{-} b^{+} c^{-} a^{+} b^{-} c^{+}
$$

\section{Cycles:}

(+) $\left(b^{+} c^{-}, c^{+} a^{-}, a^{+} b^{-}\right)$

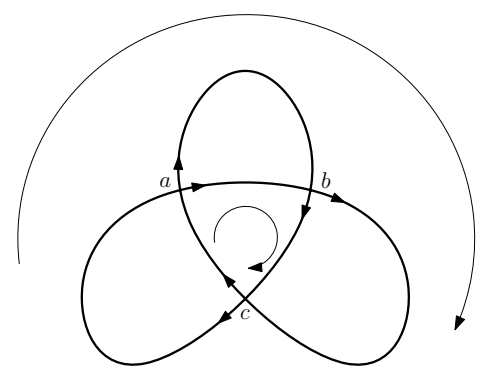

$(-)\left(a^{-} b^{+}, b^{-} c^{+}, c^{-} a^{+}\right)$External Cycle

$a^{-} a^{+} c^{-} c^{+} b^{-} b^{+}$

Cycles:

$$
\begin{aligned}
& (+)\left(a^{+} c^{-}, c^{+} b^{-}, b^{+} a^{-}\right) \\
& (-)\left(a^{-} a^{+}\right) ;\left(b^{-} b^{+}\right) ;\left(c^{-} c^{+}\right)
\end{aligned}
$$

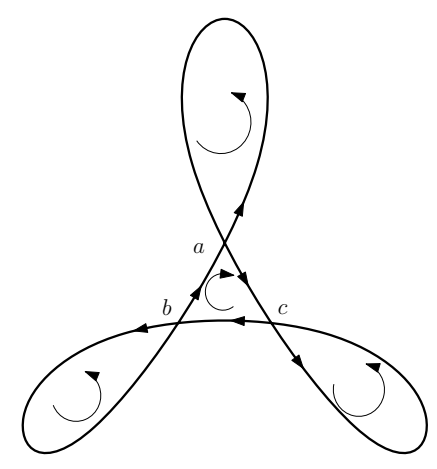

$$
a^{+} b^{-} c^{+} c^{-} b^{+} a^{-}
$$

Cycles:

$(+)\left(a^{+} b^{-}, b^{+} a^{-}\right) ;\left(c^{+} c^{-}\right)$

$(-)\left(b^{-} c^{+}, c^{-} b^{+}\right) ;\left(a^{-} a^{+}\right)$

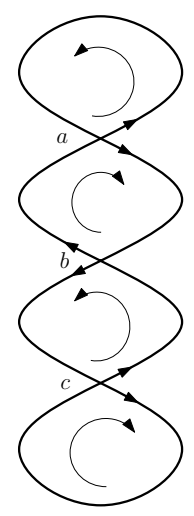


These pictures were planar views of the curves, usually it is easier to understand them. However, they might provide an exceptional phenomenon, the external cycle. It is simply a cycle in the unbounded component of the plane. What is extraordinary is that the sense of rotation of the cycle as viewed from the plane is reversed if it is external, that is, it is the opposite of what the signed word depicts.

Before we get to the requirement for a word to have a locally convex representative on its class, there is an interesting consequence of the PoincaréHopf Theorem (3) to consider. This result and its proof were provided by the author.

Proposition 3.5 Every generic oriented immersed curve on the sphere has at least 2 cycles.

Proof. Given such a curve on the sphere, it is possible to define a continuous tangent vector field $v$ with isolated zeroes by following the curve's orientation.

Close to an arc, but far from the double points, a vector field $v_{0}$ is defined via the Constant Rank Theorem. Therefore, there are no singularities in these regions.

In a neighbourhood of a double point we will have to adjust a little bit. Erase the curve near the double point and connect the branches in a way that respects the curve's original orientation and without crossings, there is only one way to do so. Now it is possible to use the Constant Rank Theorem once again to define the vector field $v_{0}$ near double points. The following image ilustrates this construction and it shows that $v_{0}$ has no singularities near the curve's double points.

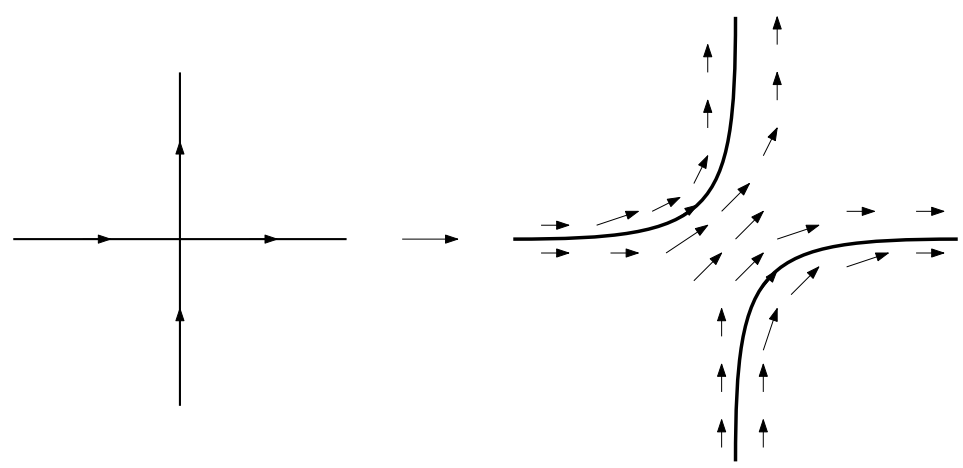

Due to the Jordan-Schönflies Theorem (4), we know that the curve divides the sphere into a finite number of connected and simply connected components, and we have already defined $v_{0}$ close to their boundary, now we must look at the interior. 
Consider such a region. Its boundary is composed of $n \geq 1$ regular arcs and $n$ double points. Topologically, they are the same as regular polygons with oriented edges. (If $n=1$ or $n=2$ we can't take regular polygons, evidently. In these cases, consider the layouts shown in the next figure).

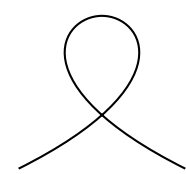

$n=1$

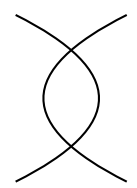

$n=2$

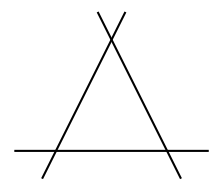

$n=3$

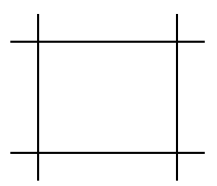

$n=4 \quad \cdots$

We will define $v$ with a single singularity in the center $O$ of the polygon. Since we have $v_{0}$ well-defined in a tubular neighbourhood of the boundary, it is defined over a closed convex curve $\sigma: \mathbb{S}^{1} \rightarrow \mathbb{S}^{2}$ next to it inside the polygon. We can define $v$ radially inside the convex region $D$ defined by $\sigma$, in the following way:

Given a point $p$ on the curve $\sigma$, consider the segment $\sigma_{p}$ going from the center of the polygon to $p$. We define $v$ using the value of $v_{0}$ over $\sigma$

$$
\sigma_{p}:[0,1] \rightarrow D \quad \sigma_{p}(0)=O \quad \sigma_{p}(1)=p
$$

$$
v\left(\sigma_{p}(t)\right)=t v_{0}(p)
$$

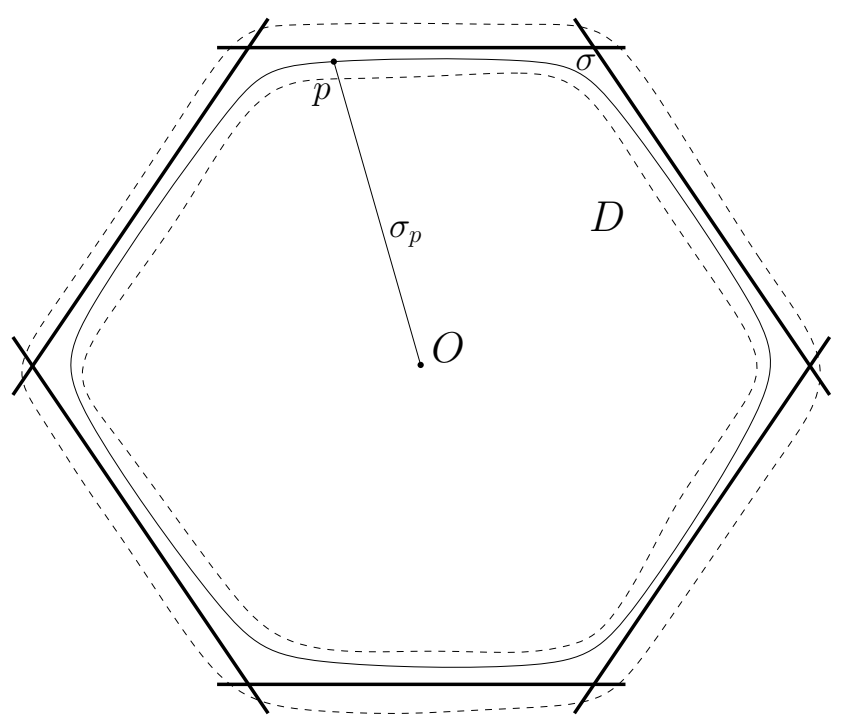

In the open set outside $\mathrm{D}$ where $v_{0}$ is defined, we take $v=v_{0}$. Thereby we have successfully defined $v$ over an open set that contains the whole connected and simply connected component. Repeating this construction for each component, we obtain the continous tangent vector field $v$ with isolated zeroes on the sphere that we wanted. 
The index $I_{i}$ of a singular point $O_{i}$ is an integer given by the degree of the map $u: \partial D \rightarrow \mathbb{S}^{1} ; u(p)=v(p) /\|v(p)\|$ where $D$ is a neighbourhood of $O_{i}$ which does not contain any other singularity, so $D$ as we have defined suits our purposes.

The Poincaré-Hopf Theorem states that if $O_{i}$ are the zeroes of $v$, then

$$
\sum_{i} I_{i}=\chi\left(\mathbb{S}^{2}\right)
$$

Since the Euler-Poincaré characteristic of the sphere is 2, the sum of the indexes of the singularities of $v$ must be 2 as well. Let us figure out these indexes.

As we saw, the singularities are in the center of polygons with $n \geq 1$ edges and vertices. We must compute it going around $\sigma$ in the positive sense since $\partial D=\sigma$. Actually, $\left.v\right|_{\sigma}$ is similar to $v$ on the border of the polygon; we just have to notice what happens near the double points.

If the consecutive edges have the same orientation, the vector field turns with the curve. For example, if the region is a cycle, $v$ makes a full rotation yielding $I_{i}=+1$, independently of the cycle's sense of rotation, and the singularity is of type center.

However, if the orientation on the edges flips, $v$ turns a fraction of $2 \pi$ in the negative sense. In general, the index depends on how many times the orientation of the edges is flipped.
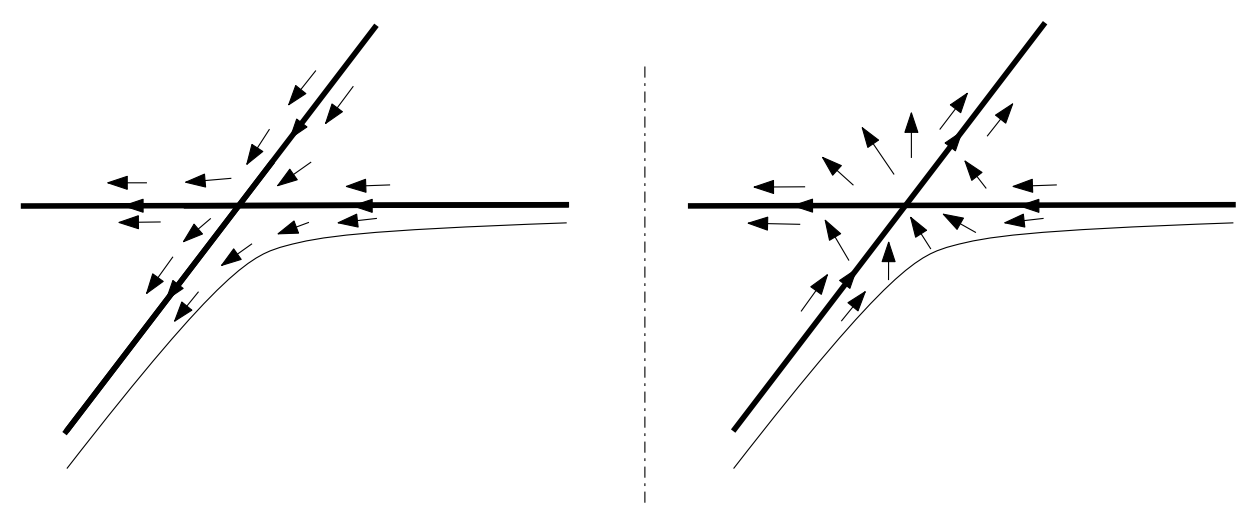

Since we can ignore the vertices where the orientation doesn't change, we end up with a regular 2 n-gon, $n \geq 1$, with alternating orientation on its edges, presuming the region is not a cycle. Now that we have reduced the situation to a very simple scenario, it is easy to see that if $n=1$, there was no need for a singularity and $I_{i}=0$. If $n \geq 2$ there are $2 n$ hyperbolic sectors and thus, by Bendixson's index formula (5) displayed below, the singularity in the center has index $I_{i}=1-n$. 
Bendixson's index formula: $I=1+\frac{e-h}{2}$

where $e$ is the number of elliptic sectors and $h$ is the number of hyperbolic sectors.

In fact, this formula holds for all cases. If the region is a cycle, then $(e=0, h=0) \Rightarrow I=1$. If $n=1$ we have a 2 -gon and $(e=0, h=2) \Rightarrow I=0$.
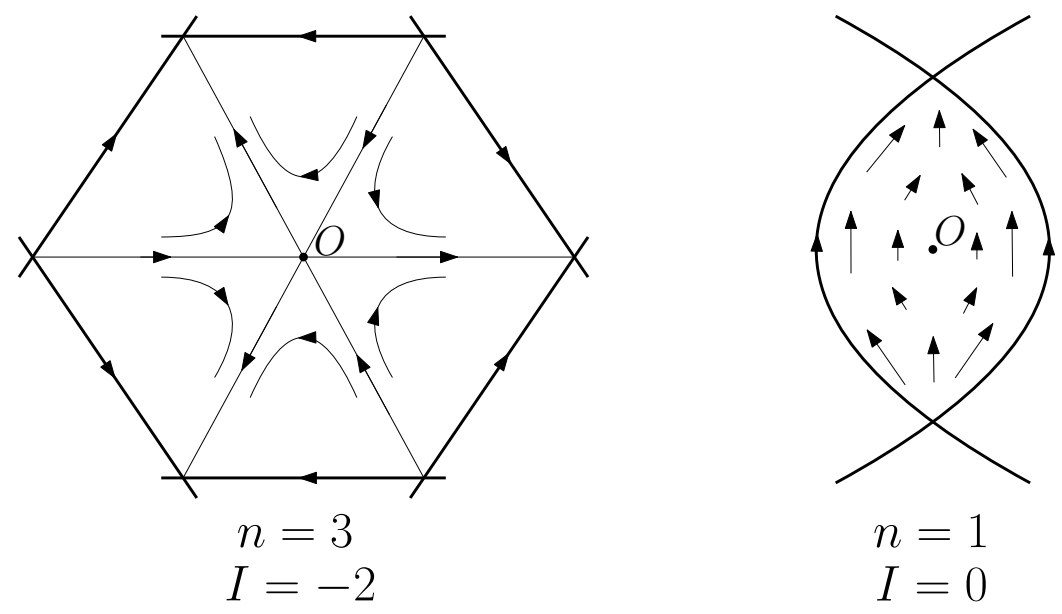

Finally, as $\sum_{i} I_{i}=2$ and only cycles contribute with positive indexes of $I_{i}=+1$, every curve must have at least 2 cycles. 


\section{4}

\section{The Words of Locally Convex Curves}

\section{1}

\section{Necessary condition on the combinatorics}

Now that we have the tools needed to study locally convex curves, we can look for further requirements on the combinatorics of their cyclic words. We present in this chapter a necessary condition on the cyclic word $w$ in order for it to have a locally convex representative in its class. This newfound requirement concerns the curve's cycles. Also, there is a clear distinction between the behaviour of these curves on the plane and on the sphere.

Since such a curve always turns in the same sense of rotation, one could suspect that all cycles should turn the same way. That is not the case if the cycle has length $n \geq 3$. The following curves are counterexamples.

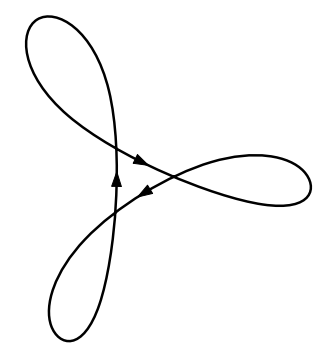

$n=3$

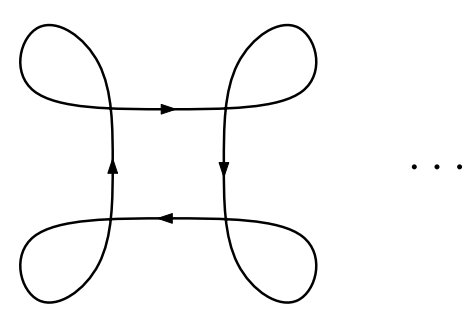

$n=4$

Nevertheless, cycles of size 1 or 2 cannot use that trick on the plane because the Gauss-Bonnet Theorem (3) doesn't allow it. Recall what it states:

Theorem 4.1 (Gauss-Bonnet) Let $R \subset S$ be a regular region of an oriented surface and let $C_{1}, \ldots, C_{n}$ be the closed, simple, piecewise regular curves which form the boundary $\partial R$ of $R$. Let $k_{g}$ denote the geodesic curvature and $K$ denote the Gaussian curvature. Suppose that each $C_{i}$ is positively oriented and let $\theta_{1}, \ldots, \theta_{p}$ be the set of all external angles of the curves $C_{1}, \ldots, C_{n}$. Then

$$
\sum_{i=1}^{n} \int_{C_{i}} k_{g}(s) d s+\iint_{R} K d \sigma+\sum_{l=1}^{p} \theta_{l}=2 \pi \chi(R)
$$

where $s$ denotes the arc length of $C_{i}$, and the integral over $C_{i}$ means the sum of the integrals in every regular arc of $C_{i}$. 
In our case, the locally convex curve delimits a cycle $R$ which is a simple region, that is, homeomorphic to a disk, so $\chi(R)=1$. Suppose $k_{g}>0$ throughout the curve and that the cycle is oriented in the opposite sense of the curve, as in the curves from the previous figure. The arcs in the boundary must be positively oriented in order to apply Gauss-Bonnet, so one must reverse the orientation of the curve, resulting in $k_{g}<0$.

If the curve is immersed in the plane, $K=0$ and the second term vanishes. Remember that we are considering the region $R$ to be simple, so this doesn't apply to external cycles. Thus we have:

$$
\sum_{l=1}^{p} \theta_{l}>2 \pi
$$

Since there is an upper bound $\theta_{l}<\pi$ on the external angles, the cycle must have length greater than or equal to 3 .

If the curve is immersed in the sphere, $K>0$, so the last argument is not enough. In fact, it is possible to have a cycle of length less than 3 with the opposite sense of rotation of a locally convex curve. However, it cannot be contained in a hemisphere, otherwise we could use the gnomonic projection to get such a cycle in a locally convex curve on the plane which would contradict Gauss-Bonnet.

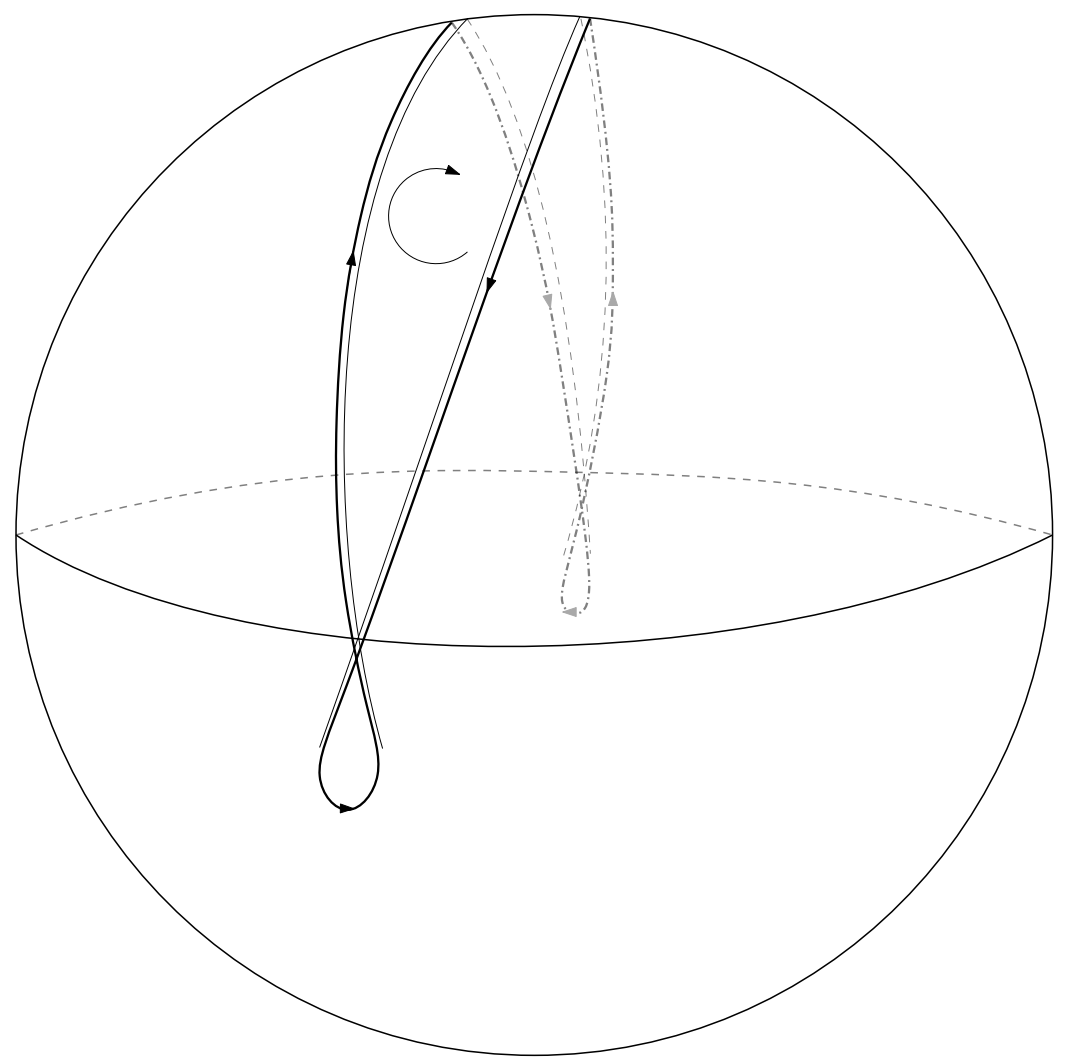

One can make the area of the cycle arbitrarily small. 
Now we just have to understand what happens if $R$ is an external cycle. In the plane, the region $R$ is unbounded and has the homotopy type of a circle, so $\chi(R)=0$. In this case, we have:

$$
\sum_{l=1}^{p} \theta_{l}=-\sum_{i=1}^{n} \int_{C_{i}} k_{g}(s) d s
$$

This equation doesn't impose restrictions on the curve; actually the sole constraint is that there can only be a single external cycle. As seen previously, its sense of rotation is the opposite to what the signed word indicates.

Finally, we can present the result. As seen above, the situation is not the same for the plane and the sphere, so we must be careful in our statements. Next we give a necessary condition on the combinatorics of a signed cyclic word for its class to have a locally convex representative on a hemisphere; equivalently, it is a necessary condition for there to exist a locally convex planar curve with that word.

Proposition 4.2 In order for a signed cyclic word $w$ to represent a locally convex curve on the plane, it cannot have two cycles with each sense of rotation and length less than or equal to 2.

Proof. Consider that $w$ has at least two cycles of length less than or equal to 2 with each sense of rotation. By picking a sense of rotation for the curve, it is not a problem to create as many cycles as needed with that same orientation. However, there are at least two that turn in the opposite sense. One can be managed as an external cycle, but another one would create a simple region $R$ that contradicts Gauss-Bonnet.

Nevertheless, this condition is not sufficient to ensure the existence of a locally convex curve on the plane for the word $w$. To see this, consider the following example:

$$
\begin{gathered}
a^{-} b^{+} c^{+} d^{-} b^{-} e^{+} d^{+} c^{-} e^{-} a^{+} \\
(+) \text {Cycles : }\left(c^{+} d^{-}, d^{+} c^{-}\right) ;\left(a^{+} a^{-}\right) \\
(-) \text {Cycles }:\left(a^{-} b^{+}, b^{-} e^{+}, e^{-} a^{+}\right)
\end{gathered}
$$

One could hope to immerse $a^{-} b^{+} b^{-} e^{+} e^{-} a^{+}$as a cycle that goes against the orientation of the curve and that isn't an exterior cycle, just as in the counterexample presented previously, but that is not possible. The only way to draw a locally convex curve on the plane with that word is if $a^{-} b^{+} b^{-} e^{+} e^{-} a^{+}$ is an external cycle. 
So if we introduce another (-)cycle of length 1 , as below, it will be impossible to create a planar locally convex curve with that word, even though it doesn't have two cycles of length less than or equal to 2 with each sense of rotation. Therefore, there is still more to learn about this subject.

$$
a^{-} b^{+} c^{+} d^{-} f^{-} f^{+} b^{-} e^{+} d^{+} c^{-} e^{-} a^{+}
$$

$(+)$ Cycles : $\left(c^{+} d^{-}, d^{+} c^{-}\right) ;\left(a^{+} a^{-}\right)$

$$
(-) \text { Cycles : }\left(a^{-} b^{+}, b^{-} e^{+}, e^{-} a^{+}\right) ;\left(f^{-} f^{+}\right)
$$
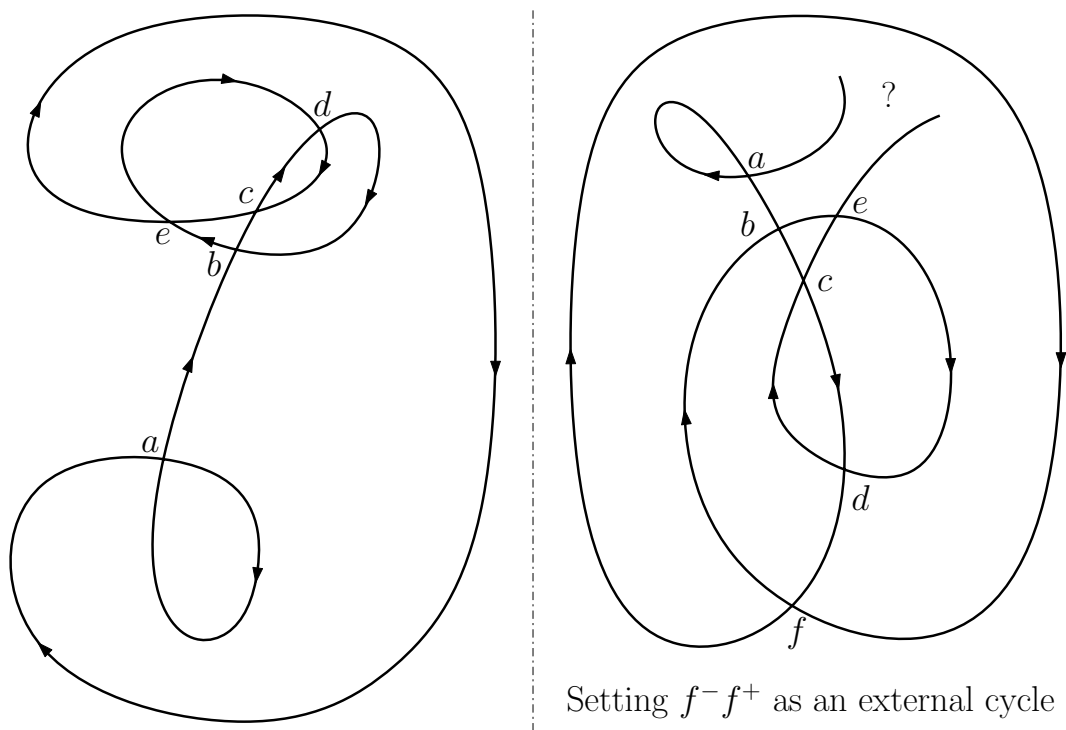

Setting $f^{-} f^{+}$as an external cycle

To conclude this chapter, it is important to clarify that it is possible to have a locally convex curve on the sphere with more than two cycles of length 1 or 2 with each sense of rotation, although it cannot fit into a single hemisphere. Just consider the following example:

$$
\begin{gathered}
a^{+} b^{-} c^{-} c^{+} d^{+} e^{-} e^{+} d^{-} f^{-} f^{+} b^{+} a^{-} \\
(+) \text {cycles : }\left(a^{+} b^{-}, b^{+} a^{-}\right) ;\left(d^{+} e^{-}, e^{+} d^{-}\right) \\
(-) \text {cycles : }\left(a^{-} a^{+}\right) ;\left(c^{-} c^{+}\right) ;\left(e^{-} e^{+}\right) ;\left(f^{-} f^{+}\right)
\end{gathered}
$$




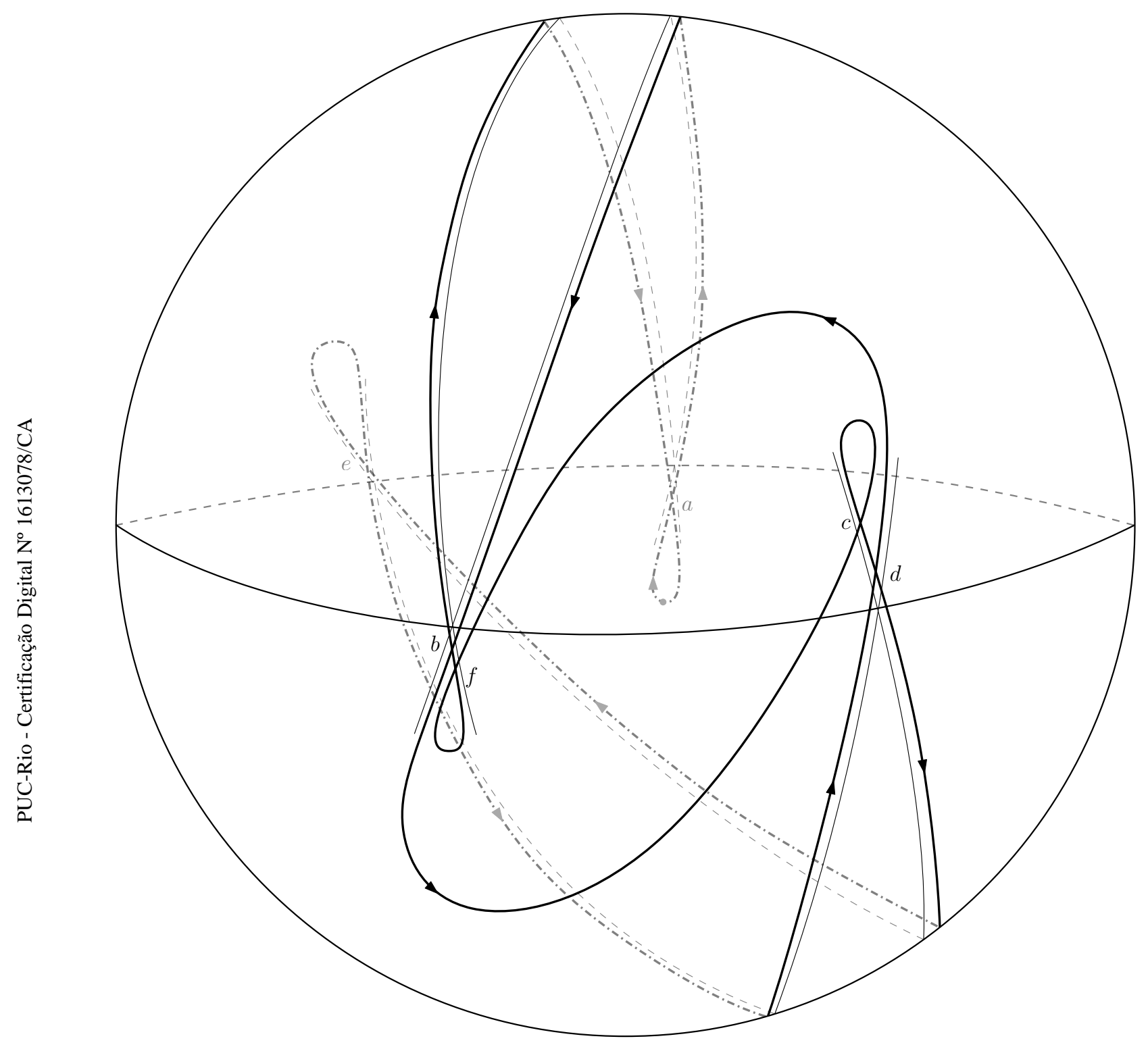




\section{5}

\section{Arnold and Whitney's Contribution}

Moving forward with the study of planar curves, there are other ways to find interesting information on their topology. The contributions of Arnold and Whitney to the matter will be presented and explored throughout this chapter.

\section{1}

\section{Arnold's generic curve invariants}

In his very rich and dense book named Topological Invariantes of Plane Curves and Caustics (2), Arnold presents us the singularity theory approach to the topological classification of mathematical objects. We will follow closely the first two sections of the said reference. The standard strategy, which goes back to Poincaré, is to consider the infinite-dimensional space $F$ of objects. In such a space there are both generic and degenerate elements. The degenerate objects form a hypersurface $\Sigma$, i.e., a codimension one subvariety which divides $F$ into parts. Each connected component of the complement of the discriminant hypersurface $\Sigma$ consists of nondegenerate objects of the same topological type, so they share the same discrete invariants.

Those invariants form the ring $H^{0}(F \backslash \Sigma)$, the zero-dimensional cohomology classes of the complement of $\Sigma$. They are locally constant functions on $F \backslash \Sigma$. One can use the natural stratification of the discriminant hypersurface according to different singularities to obtain information about the (co)homology of $\Sigma$. Subsequently, using the infinite-dimensional version of Alexander duality, one can obtain information about the (co)homology of the complement.

We shall apply this theory to the space of immersions of a circle into the plane, which we called planar curves. As previously discussed, the generic elements of this space are curves with finitely many double points and no selftangency. If two curves are in the same component of the space of immersions, there exists a one-parameter family of immersions connecting those two generic ones. However, it may be inevitable to find some degenerate elements along the way. If the path is generic, three types of events can occur: The instantaneous triple crossings of the immersion, and the instantaneous self-tangencies of two 
types; the direct self-tangencies when the two tangent branches are oriented by the same tangent vector, and the inverse self-tangencies otherwise. Here is an example of each kind of event.

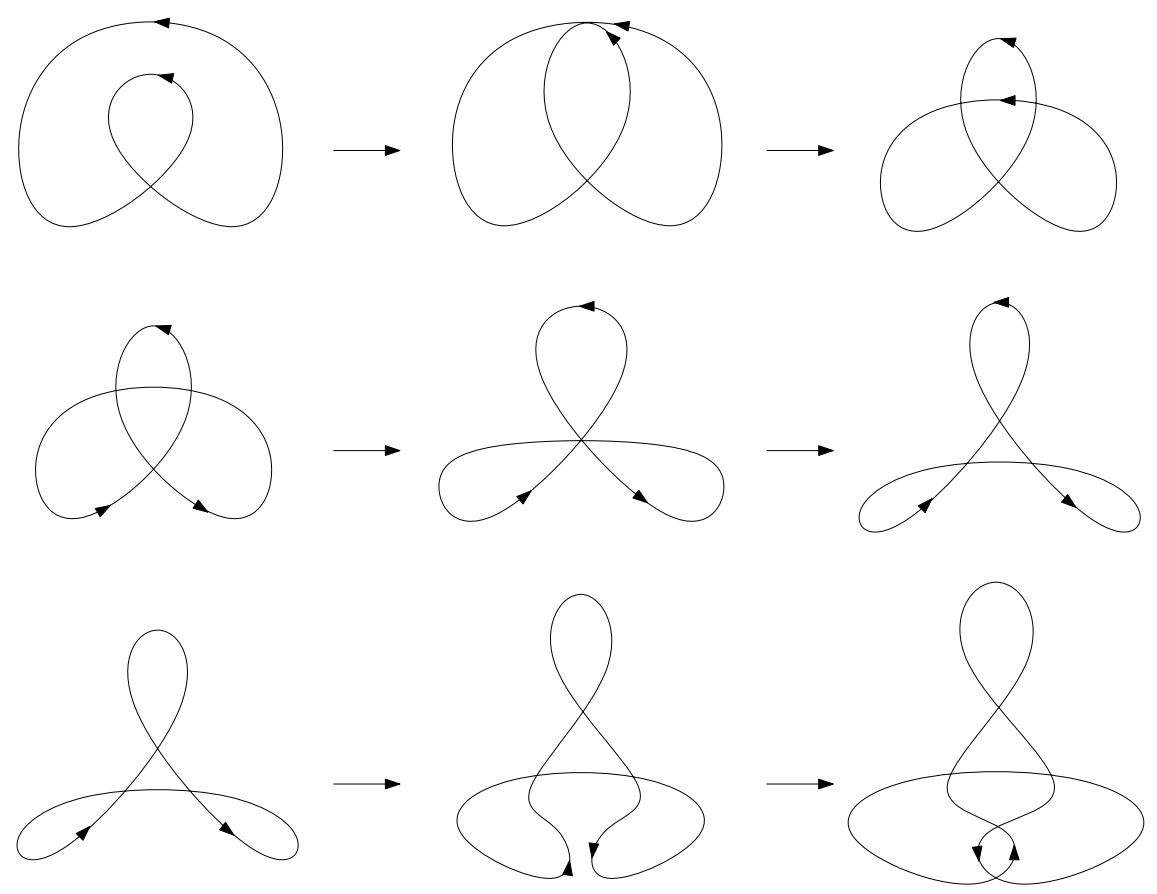

The idea is that the infinite-dimensional space of immersions of the circle into the plane has 3 hypersurfaces that divide it into many connected components of generic curves. A point in this space corresponds to a planar curve, so a path connecting two points corresponds to the one-parameter family of immersions described above. It may be that this path must cross the hypersurfaces a few times and, in fact, it is possible to define a sign to the crossings, because these hypersurfaces are cooriented.

For self-tangencies the definition is quite simple. For the direct self-tangency the crossing is positive if the number of double points increases. For the inverse self-tangency, on the other hand, the crossing is positive if the number of double points decreases.

For triple points the rule is more complicated, it is an outcome of the detailed study of the topology of codimension one singularities in the discriminant hypersurface. One must take a close look at what happens as the moving curve passes over a triple point. Close to it, there is a triangle that shrinks until it disappears and another one that is born and grows larger. We will define an orientation for the triangles which will give rise to a sign for them as well. 

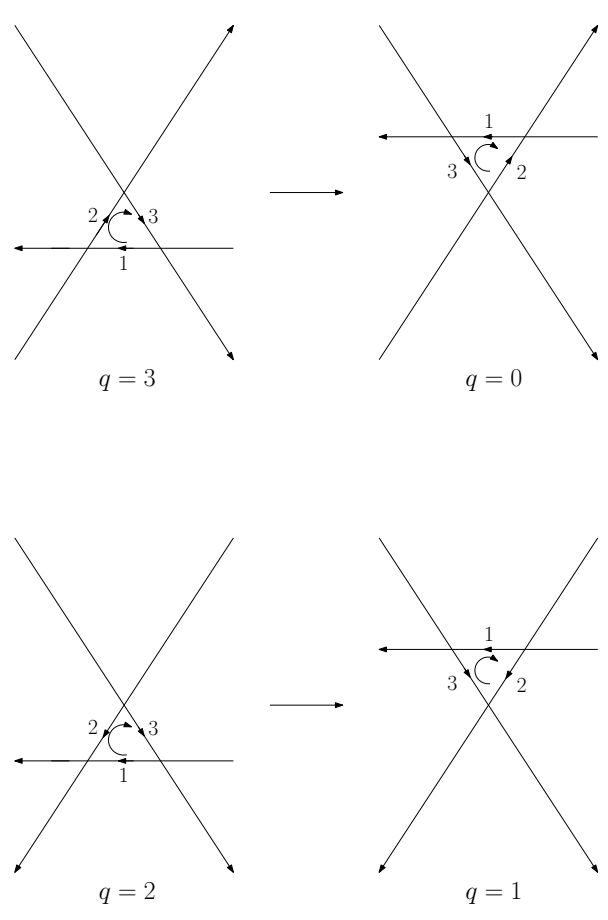

If one fixes an orientation on the curve, the three arcs that define the triangle have a well-defined cyclic order (numbered in the previous figure). So the order in which the immersed curve visits its sides defines the orientation on the face of the triangle. Each side also has its own direction, since the curve is oriented, and it may coincide with the orientation on the face or it may go against it. Let $q$ be the number of sides whose orientations coincide with that given by the cyclical order. Then we define the sign of the triangle as $(-1)^{q}$.

It is important to remark that the orientation of the curve used in the definition of the sign of the triangle is irrelevant. If one changes it, both the orientation on the face of the triangle and the orientations on the sides are flipped, so $q$ remains unchanged. Therefore, this definition works for nonoriented curves on a non-oriented plane.

Finally, it is easy to verify that the vanishing and the newborn triangles always have opposite signs. That is due to the fact that their faces share the same orientation and if an arc goes against it in one triangle, it must coincide with it in the other and vice versa. Therefore, if we add the corresponding values of $q$ for both triangles the result is always 3, which implies that they must have opposite signs.

Going back to the crossing, we say that for triple points the crossing is positive if the sign of the newborn triangle is positive (and hence the vanishing one is negative).

Arnold states that each of the three discriminant hypersurfaces may be considered as an "infinite-dimensional algebraic hypersurface", which is not 
irreducible. Actually, each of them consists of an infinite countable set of irreducible components, and they are all cooriented by the rules above. The intersection numbers with these cooriented hypersurfaces define an infinite set of invariants, but the invariants corresponding to the union of all the components of the triple points discriminant (and similarly for the two selftangency types discriminants) have nice properties and are in a sense the most basic invariants of the immersed curves.

To define the invariants, one must choose a value at one point in each path-component of the space of immersions. Thankfully, the components have been described by H. Whitney.

Theorem 5.1 (Whitney's Theorem) The space of the immersions of a circle into the plane with the same index is pathwise connected.

The index of an immersion of an oriented curve into the oriented plane is the rotation number of its tangent vector. In other words, if one considers the derivative of the immersion and normalizes it so that its target set is the unit circle $\mathbb{S}^{1}$, then the index of the immersion is the degree of the said map.

It is easy to see that if two curves are in the same path-component, they must have the same index. This is a result of the continuity of the index along the path of immersions that connects the two curves. Since the index takes values over the integers, it must be constant. To see why the converse proposition is also true, refer to Whitney's On regular closed curves in the plane (6); where there is a detailed proof.

There is a family of standard representatives of the components which are shown in the next figure. The sign of the index depends on the orientations of the curve and of the plane.

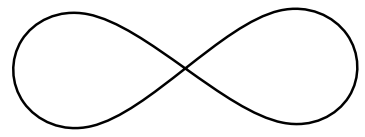

$K_{0}$

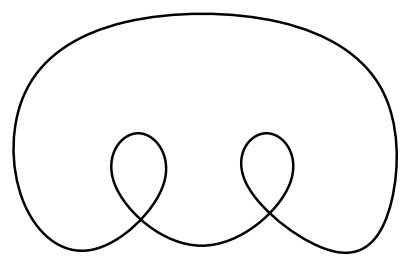

$K_{3}$

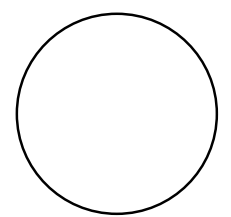

$K_{1}$

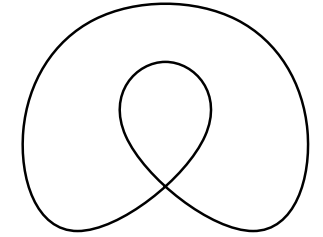

$K_{2}$

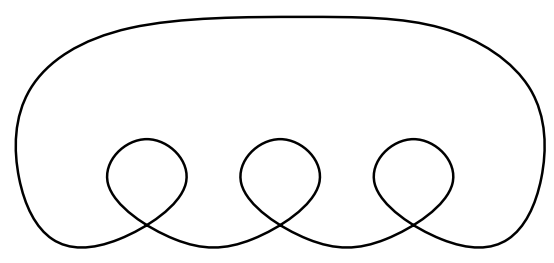

$K_{4}$ 
Now it is time to define the basic invariants $S t$ (strangeness), $J^{+}$and $J^{-}$. We just have to assign a starting value for each standard representative and choose the value $a(\cdot)$ of the jumps at positive crossings of the cooriented discriminant hypersurfaces. This is shown in the following table.

\begin{tabular}{c|ccc|cccccc} 
& $a(*)$ & $a(t)$ & $a(*)$ & & 0 & 0 & 0 & 0 & 0 \\
\hline$S t$ & 1 & 0 & 0 & 0 & 0 & 1 & 2 & 3 & $\ldots$ \\
$J^{+}$ & 0 & 2 & 0 & 0 & 0 & -2 & -4 & -6 & $\ldots$ \\
$J^{-}$ & 0 & 0 & 2 & -1 & 0 & -3 & -6 & -9 & $\ldots$ \\
\hline
\end{tabular}

These choices might seem arbitrary at first glance, but their necessity is explained by the theorems that are to come. The choice of the jumps of $J^{ \pm}$ to be 2 makes the increment of $J^{+}$equal to the increment of the number of double points and the increment of $J^{-}$is equal to the decrement of the number of double points. Since the difference $J^{+}-J^{-}$is equal to the number of double points for the standard curves $K_{n}$, it is equal to the number of double points for any curve.

Next there is a table that shows the values of the basic invariants for all immersions with two double points.

\begin{tabular}{c|c|c|c|c|c} 
& $\bigcirc$ & 0 & Q & 0 \\
\hline$|i n d|$ & 1 & 1 & 1 & 3 & 3 \\
\hline$S t$ & 0 & 0 & 1 & 2 & 3 \\
$J^{+}$ & 0 & 0 & -2 & -4 & -6 \\
$J^{-}$ & -2 & -2 & -4 & -6 & -8 \\
\hline
\end{tabular}

In order to understand the choices of constants normalizing the basic invariants, we need some new definitions first. We shall see that the invariants satisfy three properties, they are independent of the orientation of the circle, additive and local.

We already saw that the invariant $S t$ is independent of the orientation of the immersed curve and of the plane. This is also true for $J^{ \pm}$, and it is not hard to check it.

Definition 5.2 The connected sum of two immersions is defined as a new immersion obtained through the following process: place the first curve into the left half-plane and the second on the right half-plane, then connect them by embedding a bridge into the complement to their images. 


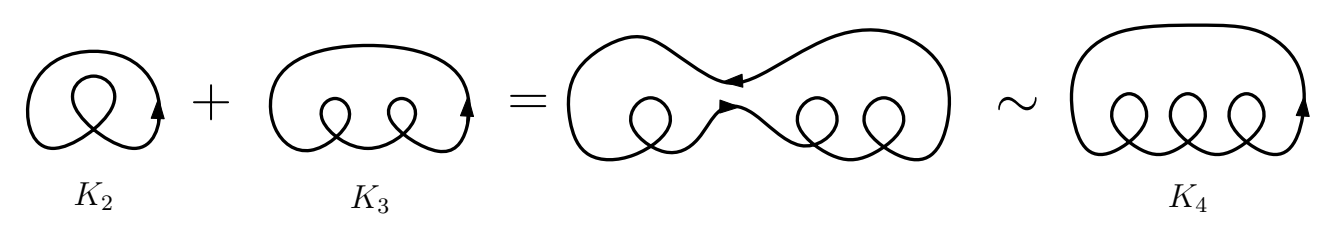

This summation is not an operation on the classes of immersions, since the bridges might be different. In fact, sometimes the sum is not even defined, which is the case for $K_{1}+K_{-1}$, because their orientations do not allow a valid embedding of the bridge. However, all three basic invariants are additive under any choice of the bridge.

For example, the basic curves $K_{n}, n \geq 2$ presented in the first table are the connected sums of the curve $K_{2}$.

$$
\begin{gathered}
K_{i+1}=i K_{2} \quad(i>0) \\
K_{i+1}+K_{j+1}=K_{i+j+1} \quad(i, j \geq 0)
\end{gathered}
$$

Definition 5.3 An invariant of a nondegenerate immersion is called local if its increments under a generic crossing of the corresponding discriminant hypersurface depends only on the behaviour of the family of immersions in the neighbourhood of the singularity.

Now we can formulate the axiomatic definitions of the basic invariants, explaining the choices of the normalizing constants.

Theorem 5.4 There exists one and only one invariant (St) related to triple points of immersions of the circle into the plane that satisfies the following axioms:

(1) It is local;

(2) It is additive;

(3) It is independent of the orientation of the circle;

These three properties determine the invariant uniquely up to a multiplicative constant, which is fixed by the last condition.

(4) $\operatorname{St}\left(K_{2}\right)=1$.

Similar theorems hold for the other two basic invariants, switching the triple points for direct tangencies or inverse tangencies, and changing the value of the fixed initial constant.

As an application of this theory, here is a third proof of the claim first presented in chapter two: A generic immersed closed planar curve with $n$ double points divides the plane into exactly $n+2$ connected components. First we 
check that the formula holds for all standard curves $K_{i}$ that represent the components of the space of immersions. Now it suffices to study what happens when one crosses the discriminant hypersurfaces, that is, take a close look at the number of double points and regions when the value of $S t, J^{+}$or $J^{-}$ changes.

The easiest one is the $S t$ invariant, because both the number of double points and regions remain the same when a curve passes over a triple point.

For $J^{+}$and $J^{-}$, consider the following picture.

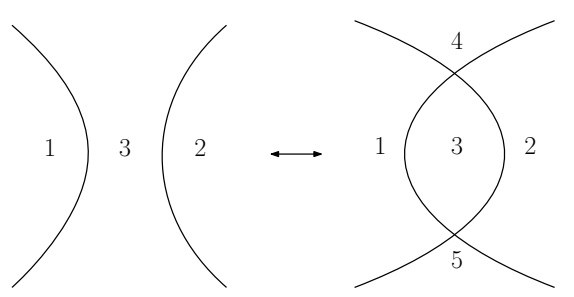

The orientation here doesn't matter, so the argument is the same for both cases, we just have to see how many new regions there are when we create two new double points. As shown in the last figure, the region labelled 3 gets split into three regions, so we get two new ones. The only thing we have to verify is that regions 4 and 5 are truly two distinct regions of the plane. We have faced a similar problem in the second proof in chapter 2, and we shall apply the same reasoning here.

Suppose that 4 and 5 are the same region on the plane, then there is a path $\mu$ that does not cross the original curve going from 4 to 5 . If that is the case, then before the $J^{ \pm}$crossing, there was a closed curve $\mu^{\prime}$ separating two portions of the original curve. By the Jordan Curve Theorem the original curve cannot be connected, which is a contradiction. So 4 and 5 are indeed two new regions and the formula holds after one crosses the discriminant hypersurface. This implies that any generic planar curve with $n$ double points divides the plane into exactly $n+2$ connected components, which concludes the proof.

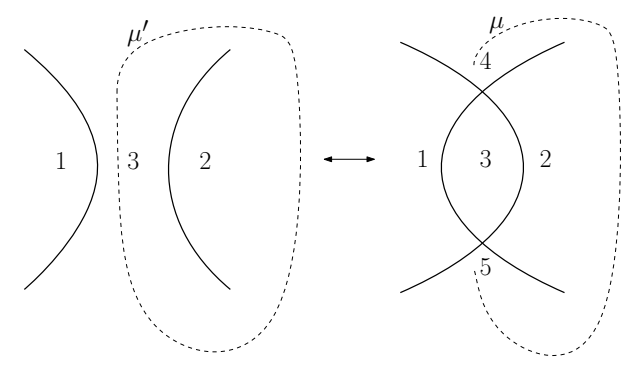




\section{2}

\section{Whitney's formula for double points}

In his article, besides proving his aforementioned theorem, Whitney also presents a formula relating the index of a curve with its double points. In this section we'll investigate the concepts dealing with it and provide a new proof.

First we need some new definitions to make sense of Whitney's formula. Consider a generic immersed closed planar curve $\gamma$. Setting a regular point $p$ on $\gamma$ as the starting point one can pick a direction and go around the curve. Under these circumstances, we call $\gamma$ an oriented pointed curve with base point $p$. Also, if $p$ belongs to the border of the unbounded component of the plane, we say that $\gamma$ has an outside starting point.

Now, one can associate a sign to each double point. To do so, Whitney has chosen the opposite orientation to the one we have considered thus far. In chapter 1 we gave a sign to each crossing of the double point according to the orientation of the plane, assigning + to "the first one" and - to "the second". Departing from $p$ we pass twice over a double point $a_{i}$, either in the order $a_{i}^{+} a_{i}^{-}$or $a_{i}^{-} a_{i}^{+}$. Whitney defined that the double point is positive if the order is $(-,+)$ and it is negative if the order is $(+,-)$. Notice that the sign of a double point depends on the choice of the starting point $p$, and if the orientation of the curve is inverted, the sign of the double point also changes.

Given an oriented pointed curve $\gamma$, one can count the number of positive double points $\left(N^{+}\right)$and the number of negative ones $\left(N^{-}\right)$. Finally, we can state the theorem that contains Whitney's formula.

Theorem 5.5 If $\gamma$ is an oriented pointed curve with an outside starting point $p$, then

$$
\operatorname{ind}(\gamma)=N^{+}-N^{-}+\mu
$$

where $\mu=1$ if the orientation of the curve sets the unbounded component of the plane to the right of $p$, and $\mu=-1$ if it is to the left of $p$.

The simplest interpretation of this formula is to consider that an oriented pointed curve with an outside starting point with the unbounded component to its right "starts" with index 1 . To see this just consider the oriented circle. Now imagine that the positive double points contribute with positive turns and the negative double points add negative turns, which can easily be seen in the following example. However, it might not be clear yet for an arbitrary curve. 


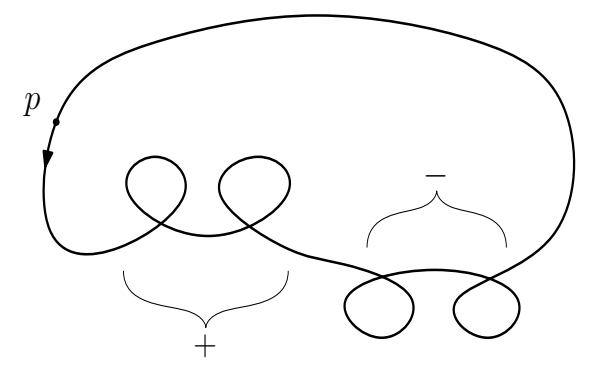

Here is a new proof for the theorem with a more geometric flavour. We just have to reduce it to simpler cases by taking the double points away one by one, so the proof is by induction.

Proof. Let $\gamma$ be an oriented pointed curve with an outside starting point with the unbounded component to its right, the other case is analogous. The base case is the circle, which has no double point and trivially satisfies the formula. For a curve $\gamma$ with $n$ double points, start at $p$ and go around the curve until the first double point is reached. One can delete it and reconnect the four branches in its neighbourhood without crossings in a way that disconnects the curve, as in the figure below.

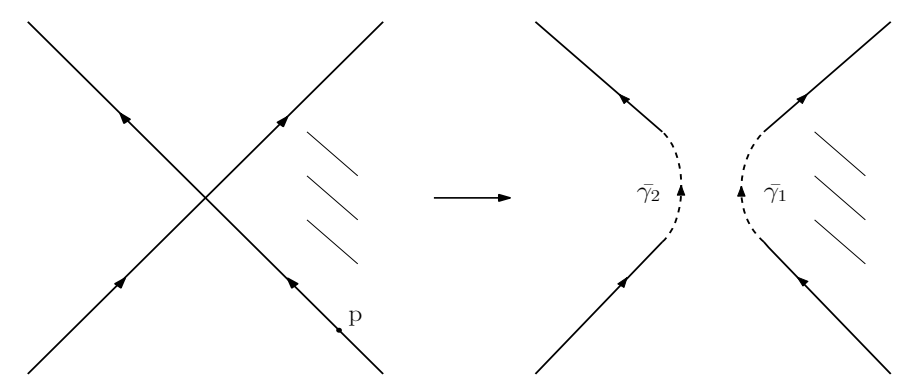

Now there are two curves $\gamma_{1}$ and $\gamma_{2}$ and the following relation: $\operatorname{ind}(\gamma)=\operatorname{ind}\left(\gamma_{1}\right)+\operatorname{ind}\left(\gamma_{2}\right)$, since the two small dashed arcs $\overline{\gamma_{1}}$ and $\overline{\gamma_{2}}$ that were introduced have opposite contributions to the index. The other double points of the original curve are divided into three classes. They can be double points of $\gamma_{1}$, double points of $\gamma_{2}$, or intersections of those two curves.

In chapter 1 we saw that two generic curves must intersect at an even number of points $(2 k)$, and in fact they split evenly between positive and negative double points in the original curve $\gamma$. To see why, consider a generic translation of $\gamma_{2}$ until there are no more intersections with $\gamma_{1}$. If we hadn't removed the first double point, such movement would represent a generic path of immersions, so we can apply Arnold's theory of invariants. One can verify that the triple point crossings preserve the signs of the double points, and the 
self-tangencies can create or remove two double points, but always of opposing signs. As the $2 k$ intersections between $\gamma_{1}$ and $\gamma_{2}$ are the only ones to disappear, one concludes that half of them must have been positive and the other half must have been negative in $\gamma$.

Before we apply the induction step, the problem splits into two cases depending on the sign of the removed double point.

If it was positive, then we set points $p_{1}$ and $p_{2}$ on $\overline{\gamma_{1}}$ and $\overline{\gamma_{2}}$ as base points for $\gamma_{1}$ and $\gamma_{2}$, respectively.

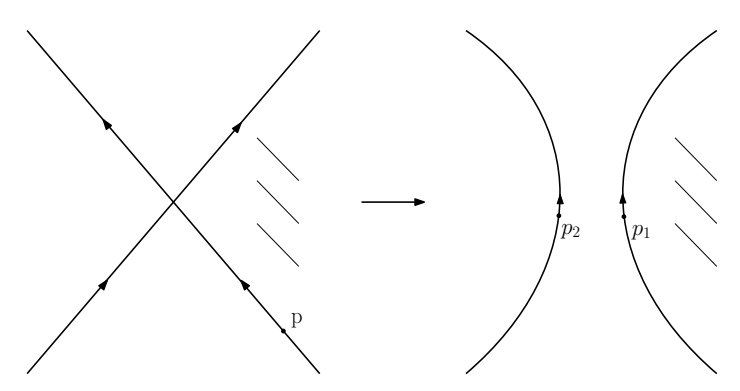

Considering them individually, both are oriented pointed curves with an outside starting point with the unbounded component to the right, so we apply the induction step.

$$
\begin{aligned}
& \operatorname{ind}\left(\gamma_{1}\right)=N_{1}^{+}-N_{1}^{-}+1 \\
& \operatorname{ind}\left(\gamma_{2}\right)=N_{2}^{+}-N_{2}^{-}+1
\end{aligned}
$$

Since a positive double point was removed from $\gamma$, we have:

$$
\begin{aligned}
& N^{+}=N_{1}^{+}+N_{2}^{+}+1+k \\
& N^{-}=N_{1}^{-}+N_{2}^{-}+k
\end{aligned}
$$

So we derive:

$$
\begin{aligned}
N^{+}-N^{-}= & \left(N_{1}^{+}+N_{2}^{+}+1+k\right)-\left(N_{1}^{-}+N_{2}^{-}+k\right) \\
= & \left(N_{1}^{+}-N_{1}^{-}\right)+\left(N_{2}^{+}-N_{2}^{-}\right)+1 \\
= & \left(i n d\left(\gamma_{1}\right)-1\right)+\left(i n d\left(\gamma_{2}\right)-1\right)+1 \\
= & i n d(\gamma)-1 \\
& \operatorname{ind}(\gamma)=N^{+}-N^{-}+1
\end{aligned}
$$


If the removed double point was negative, we do the same for the base points, but this time $\gamma_{2}$ has the unbounded component to its left.

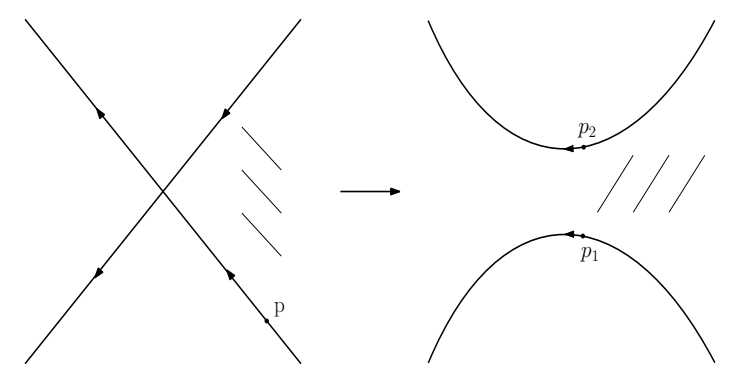

The calculation is similar.

$$
\begin{aligned}
& \operatorname{ind}\left(\gamma_{1}\right)=N_{1}^{+}-N_{1}^{-}+1 \\
& \operatorname{ind}\left(\gamma_{2}\right)=N_{2}^{+}-N_{2}^{-}-1
\end{aligned}
$$

$$
\begin{aligned}
& N^{+}=N_{1}^{+}+N_{2}^{+}+k \\
& N^{-}=N_{1}^{-}+N_{2}^{-}+1+k \\
N^{+}-N^{-}= & \left(N_{1}^{+}+N_{2}^{+}+k\right)-\left(N_{1}^{-}+N_{2}^{-}+1+k\right) \\
= & \left(N_{1}^{+}-N_{1}^{-}\right)+\left(N_{2}^{+}-N_{2}^{-}\right)-1 \\
= & \left(\operatorname{ind}\left(\gamma_{1}\right)-1\right)+\left(\operatorname{ind}\left(\gamma_{2}\right)+1\right)-1 \\
= & \operatorname{ind}(\gamma)-1 \\
& \\
& \operatorname{ind}(\gamma)=N^{+}-N^{-}+1
\end{aligned}
$$

This concludes the proof.

The study of Whitney's formula traversed the twentieth century. Arnold himself has worked on it, and he has mentioned in his book from 1994 (2):

"As far as I know, this Whitney formula, proved in 1937, has not yet been absorbed by modern mathematics, and its higher-dimensional generalizations are still to be found".

Fortunately, research did not stop there. The work of a contemporary mathematician stands out, the many papers of Michael Polyak and his coauthors. They have successfully generalized Whitney's formula in several ways. 
One can define an index of a point with respect to a curve to extend the result to other situations (7), there are generalizations for oriented punctured surfaces (8), new formulas have been found for higher dimensions (9), and even relations with Arnold's invariant St were deduced. So one can say that the subject is much better understood nowadays and it is still rich and active in the twenty-first century. 


\section{6}

\section{Bjerre and Double Tangents}

So far the double points of generic immersions of $\mathbb{S}^{1}$ into the plane have been addressed from multiple viewpoints, but we have not given any thought to its double tangents yet. A double tangent is a line on the plane that is tangent to an immersed curve at two distinct points. In order to better understand their properties, we will look into some papers on the subject written by FabriciusBjerre (10),(11),(12).

\section{1}

\section{Relating double points, double tangents and inflectional points}

First of all, it is important to point out that just as with double points, a generic curve on the plane can have some double tangents, but triple tangents are not generic, like triple points. If one moves the curve slightly, a triple tangent can be undone. Furthermore, the points of inflection of the curve will play a special role, so we will assume that no double tangent is tangent at such a point. This phenomenon is also singular; it represents a higher contact order between the curve and the tangent line. It is good to highlight that any generic closed curve must have an even number of inflectional points, since they represent a change in the sign of the curve's concavity.

We need some terminology for the double tangents. Consider a double tangent $r$ whose points of contact with the curve are $R_{1}$ and $R_{2}$. The convex arcs in the neighbourhood of those points can be either on the same half-plane defined by $r$ or not. If they are on the same side, we call $r$ an exterior double tangent, otherwise it is called an interior double tangent.

The tangent line can be divided into two rays with initial point at the point of contact. If the curve is oriented, one can give signs to them, appointing as the positive half-tangent the ray that points at the same direction as the tangent vector of the curve. Naturally, the other ray is called the negative half-tangent.

At a double tangent, the positive half-tangents at $R_{1}$ and $R_{2}$ may point the same way or at opposite directions, in which case they might be one against the other or they might point away from one another. So there is a total of six types of double tangents named $E_{1}, E_{2}, E_{3}, I_{1}, I_{2}, I_{3}$ which are explained in 
the following figure. Notice that if we change the orientation of the curve, $E_{2}$ and $E_{3}$ interchange, as well as $I_{2}$ and $I_{3}$, while $E_{1}$ and $I_{1}$ stay invariant.

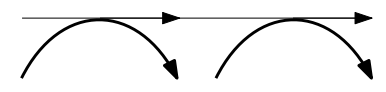

$E_{1}$

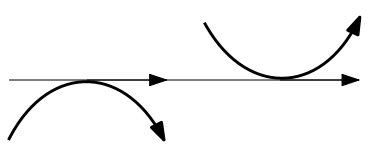

$I_{1}$

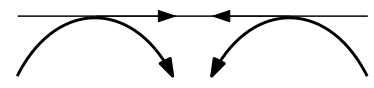

$E_{2}$

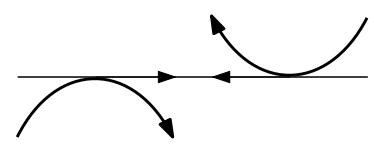

$I_{2}$

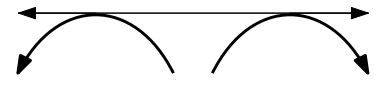

$E_{3}$

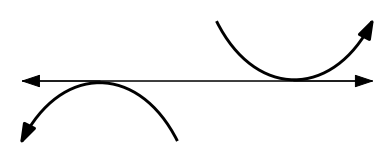

$I_{3}$

Bjerre uses these definitions to attain a formula relating the number of exterior double tangents $(t)$, interior double tangents $(s)$, double points $(d)$ and inflectional points $(2 i)$, which is:

$$
t-s=d+i
$$

Here is the proof presented in his paper On the Double Tangents of Plane Closed Curves (10). Consider a generic closed curve on the plane $\gamma$. We will count the number of points of intersection of the half-tangents and $\gamma$ as a base point $P$ traverses the curve. Let $p$ denote the tangent line at $P$, and we will call $p+$ the positive half-tangent and $p-$ the negative half-tangent. The number of intersections remains unchanged unless $P$ passes a double point or an inflectional point, or if the tangent $p$ passes a double tangent.

If $P$ passes a double point, a common point of $p+$ and $\gamma$ is lost and it becomes a common point of $p$ - and $\gamma$. The same happens when $P$ passes over a inflectional point.

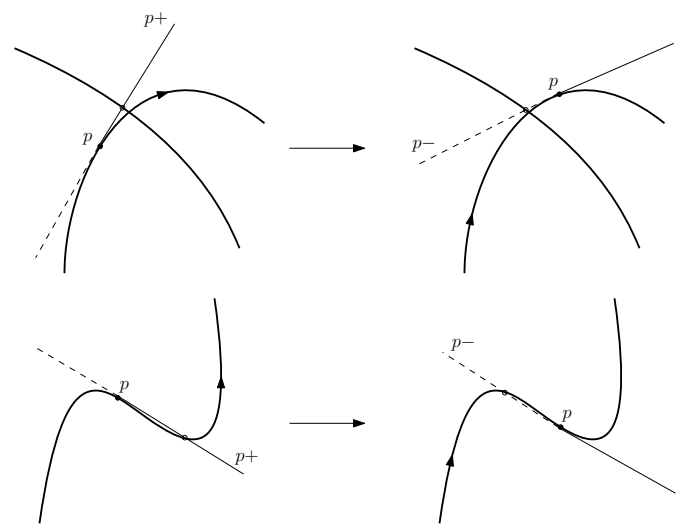


When $p$ passes a double tangent, its type determines the event. For $E_{1}$ and $I_{1}$, the points of contact $R_{1}$ and $R_{2}$ are not symmetrical, in the sense that $p+$ contains the other point in one passing, but not in the other. At the passing in which $p+$ contains the other point, it gains two new intersection points in the case of $E_{1}$ and loses two points in the case of $I_{1}$. At the other passing it is $p$ - that loses two points at $E_{1}$ and gains two points at $I_{1}$.

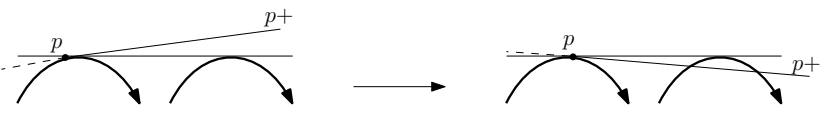

$E_{1}$
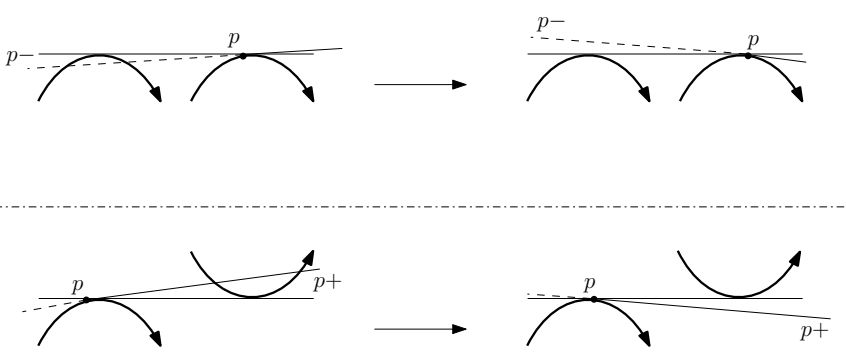

$I_{1}$
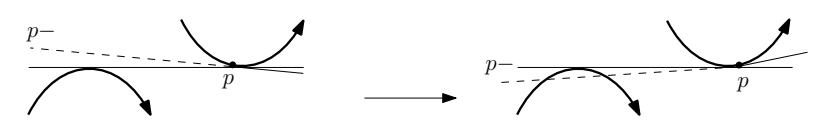

For $E_{2}$ and $I_{2}$, only $p+$ is affected. At each passing it gains two points at $E_{2}$ and it loses two points at $I_{2}$. Finally, for $E_{3}$ and $I_{3}$ similar events happen to $p$-. At each passing it loses two points at $E_{3}$ and it gains two points at $I_{3}$. These statements are intuitively clear, but exact proofs can also be given.
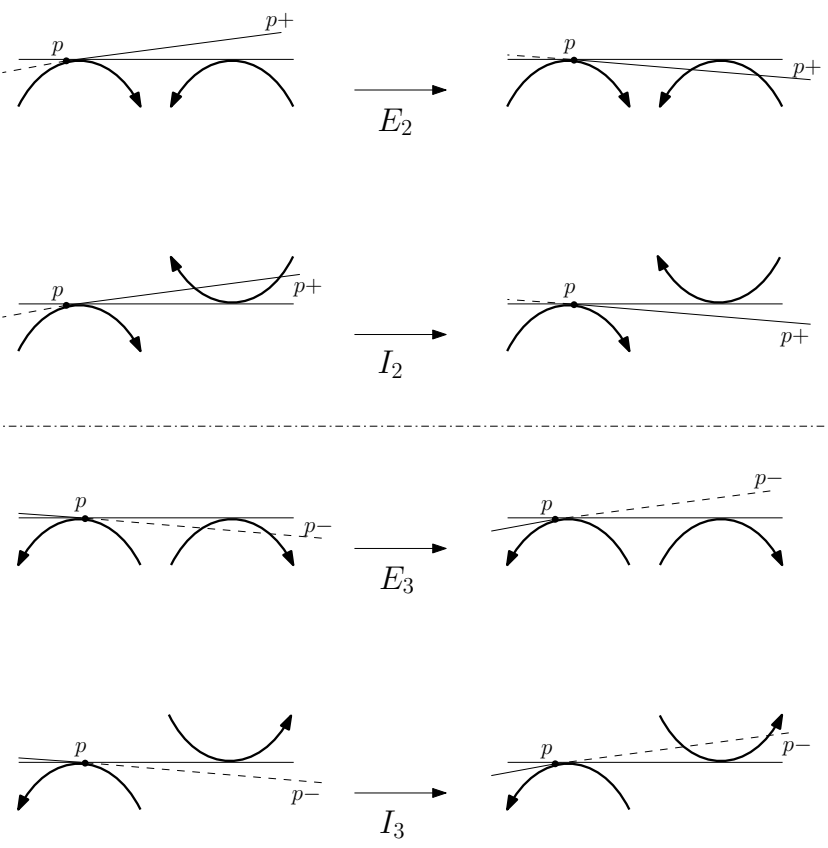
If we imagine $P$ going around $\gamma$ once, we can count the number of points gained and lost both for $p+$ and $p-$. The total number of intersection points must be the same at the start and at the end of the lap. Consider $t_{1}, t_{2}, t_{3}$ as the numbers of exterior double tangents of types $E_{1}, E_{2}, E_{3}$ respectively, and $s_{1}, s_{2}, s_{3}$ as the numbers of interior double tangents of types $I_{1}, I_{2}, I_{3}$ respectively. Recall that the curve has $d$ double points and $2 i$ inflectional points.

The previous analysis shows that $p+$ gains $2 t_{1}+4 t_{2}$ points and it loses $2 s_{1}+4 s_{2}+2 d+2 i$, while $p-$ gains $2 s_{1}+4 s_{3}+2 d+2 i$ points and it loses $2 t_{1}+4 t_{3}$ points. Therefore, we have the following equations:

$$
\begin{aligned}
& 2 t_{1}+4 t_{2}=2 s_{1}+4 s_{2}+2 d+2 i \\
& 2 t_{1}+4 t_{3}=2 s_{1}+4 s_{3}+2 d+2 i
\end{aligned}
$$

Adding both equation we obtain:

$$
\begin{gathered}
4\left(t_{1}+t_{2}+t_{3}\right)-4\left(s_{1}+s_{2}+s_{3}\right)=4 d+4 i \\
t-s=d+i
\end{gathered}
$$

Here is the Theorem in Bjerre's words:

Theorem 6.1 For the closed curve $\gamma$, the difference between the number of exterior and interior double tangents is equal to the sum of the number of double points and half the number of inflectional points. Hence, the number of exterior double tangents is greater than or equal to this sum. Equality holds if and only if $\gamma$ has no interior double tangents.

One can also consider special cases, such as for locally convex curves, which have no inflectional points. A direct consequence of the theorem is that: Corollary 6.2 A locally convex curve $\gamma$ has at least as many double tangents as it has double points, since $i=0 \Rightarrow t-s=d$.

In fact, there is a special family of locally convex curves whose members have the minimum number of double tangents, i.e., $s=0$, and thus the formula for this family is $t=d$. This special set of curves will be studied later on.

Another fine result relates the index $i n d(\gamma)=v$ of a curve with the number of double tangents.

Corollary 6.3 A curve $\gamma$ with index $v=0$ has at least two double tangents. A curve $\gamma$ with index $v \geq 1$ has at least $v-1$ double tangents. Any curve that attains this bound is necessarily locally convex, i.e., if it is not locally convex, then the lower bound is $v$. 
Proof. We must recall Whitney's formula: $v=N^{+}-N^{-} \pm 1$. If $v=0$ we have

$$
N^{+}-N^{-}= \pm 1
$$

So this implies that $\gamma$ must have at least one double point. Furthermore, a curve with index 0 must have at least two inflectional points. Since $d \geq 1$ and $i \geq 1$, we have that $t-s \geq 2$, which implies that the curve must have at least two double tangents.

If $v \geq 1$ Whitney's formula provides that $v \pm 1=N^{+}-N^{-}$, and thus $d \geq v-1$. Bjerre's theorem gives us that $t-s \geq v-1+i$, so the original statement is true and $\gamma$ can only have $v-1$ double tangents if $i=0$, that is, if it is locally convex.

\section{2}

\section{The special family $\mathfrak{B}$}

Now we will consider a special family of curves, let's call it $\mathfrak{B}$ as a tribute to Bjerre, who has presented and analysed it in his paper On a class of locally convex closed curves (11). For a generic curve $\gamma$ to be in this set, it must be locally convex and there must exist in the plane a line $u$ and a point $U^{\prime}$ not on $u$, such that $\gamma$ has no points in common with $u$ and possesses no tangent through $U^{\prime}$.

Any curve $\gamma \in \mathfrak{B}$ defines two regions in the plane, namely the Exterior Domain $(\omega)$ and the Kernel $\left(\omega^{\prime}\right)$.

The exterior domain is an open set in the unbounded component of the plane. It is the complement of the convex hull of the set of points in $\gamma$. We will denote its boundary by $h$; it consists of parts of the most external arcs of $\gamma$, i.e., those that border the unbounded component of the plane, and segments of double tangents. The totality of lines $u$ that have no intersection with $\gamma$ fill up the exterior domain $\omega$, and if the curve is convex, then it coincides with $h$.

The kernel is a dual to the exterior domain. It consists of the totality of points $U^{\prime}$ through which no tangent of $\gamma$ passes. Its boundary is a convex curve $h^{\prime}$ composed of the innermost arcs of $\gamma$, joined one to another by some double points. Again, if the curve is convex, it also coincides with the boundary of the kernel $h^{\prime}$

Therefore, the curve $\gamma$ lies in a ring-shaped domain between the exterior domain and the kernel which is bounded by $h$ and $h^{\prime}$. Since $\gamma$ is locally convex and one cannot draw tangent lines to it from points in the kernel, it follows that $\gamma$ is always concave towards the kernel $\omega^{\prime}$ and it is always convex towards the exterior domain $\omega$. Here are some examples of curves $\gamma \in \mathfrak{B}$. 

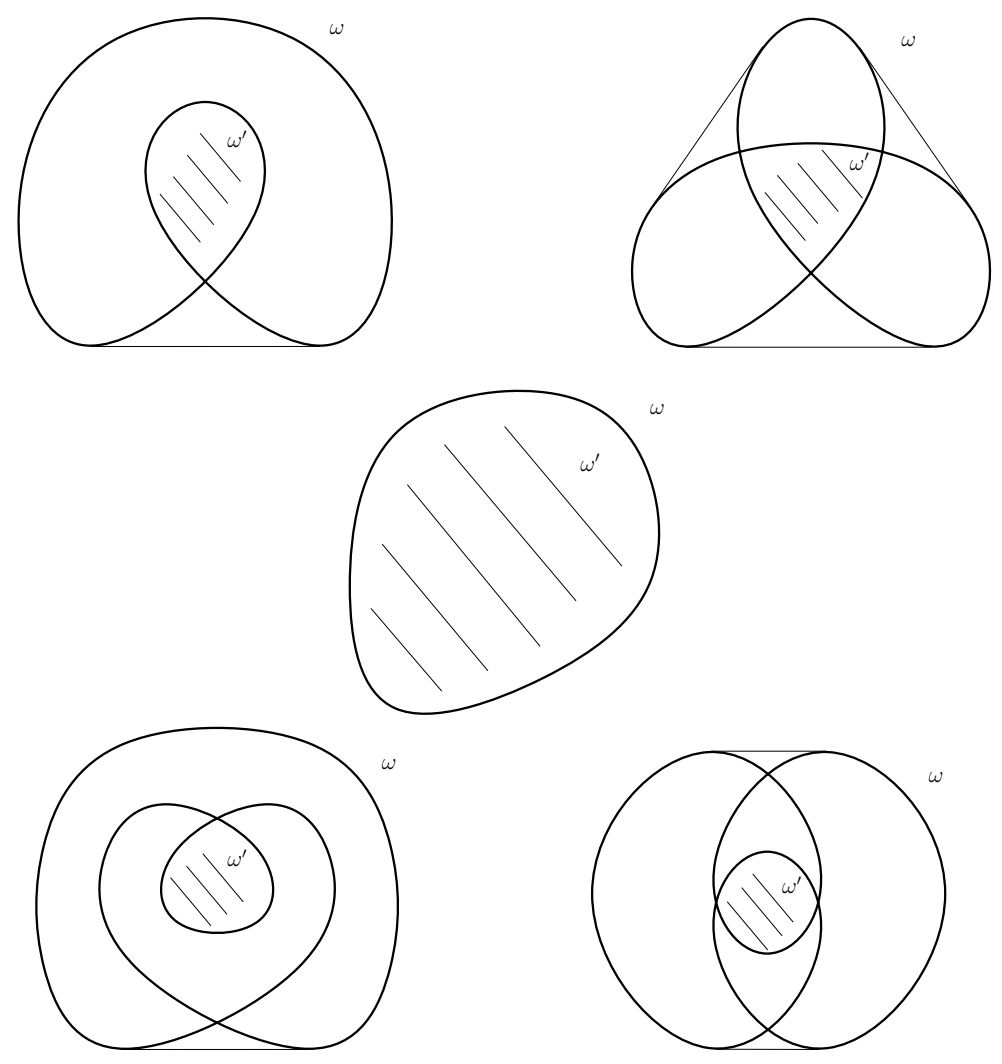

As previously stated, the curves in this family have no interior double tangents. To see why, suppose there was such a double tangent. It cannot go through the kernel $\omega^{\prime}$, so it must look like this:

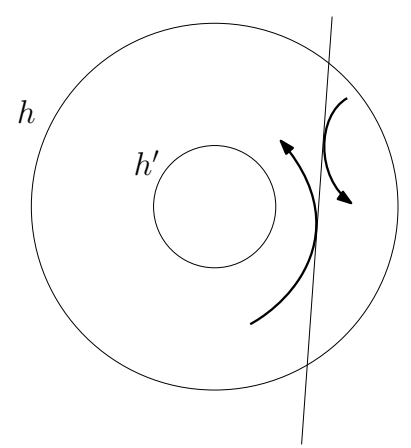

Therefore, there is a portion of the curve that is concave towards the exterior domain, which is impossible.

The curve in the family $\mathfrak{B}$ must go around the kernel remaining inside the ring-shaped domain. Traversing the curve, one gets "closer" to the kernel or "moves away" from it. This can be noticed when crossing the double points, because when we cross the curve from the convex to the concave side we approach the kernel, whereas the opposite happens when we get closer to $h$.

Using this fact, Bjerre defines a new way to give signs to the double points. Starting from a point $H$ on the curve situated at the boundary of the 
exterior domain, we say that a double point $D$ is positive if the first time we cross it we get closer to the kernel. On the other hand, $D$ is negative if the first time we pass it, we go from the concave to the convex side of $\gamma$.

The partition between positive and negative double points is independent of the orientation of the curve. Although the sign of a particular double point depends on the choice of $H$, the total number of positive and negative double points remain invariant.

Since the curve must always be concave towards the kernel, this orientation given by Bjerre actually coincides with the orientation defined by Whitney if the curve turns counter-clockwise. Every time we approach the kernel, the tangent vector at $D$ is to the left of the tangent vector of the second passing, so if $D$ is positive to Bjerre, it is also positive according to Whitney. The same reasoning applies for the negative double points. However, if $\gamma$ turns clockwise, Bjerre's and Whitney's orientations disagree, they are the opposite of one another.
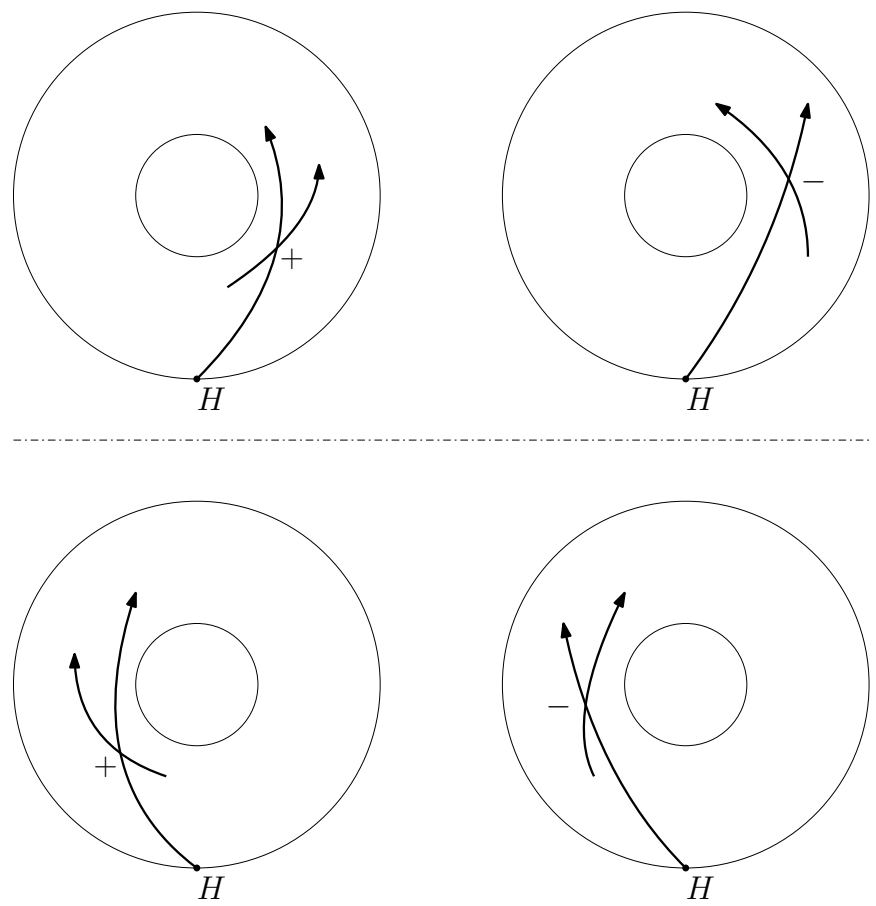

It is not surprising, therefore, that Bjerre derives the formula

$$
k_{1}-k_{2}=|v|-1
$$

where, according to his orientation, $k_{1}$ is the number of positive double points, $k_{2}$ is the number of negative double points and $v$ is the index of $\gamma$. The absolute value of $v$ appears on the formula in order to correct the difference in the signs for clockwise oriented curves. Bjerre mentions the work of Whitney in 
his paper, but it is fruitful to have a new proof, even if it applies only to a restricted class of curves.

Furthermore, he also assigns signs to the double tangents and arrives at a corresponding formula for them. The definition of the sign is dual to the previous definition for double points. We shall begin at a point $H^{\prime}$ in $h^{\prime}$ and go around the curve. Let $P$ be the traversing point and $p$ the tangent to $\gamma$ at that point. A double tangent $d$ is called positive if $p$ loses two points of intersection with $\gamma$ at the first passage, and hence it gains two points of intersection at the second time it passes $d$. If that occurs in the opposite order, then $d$ is a negative double tangent.

Again, this partition does not depend on the orientation of the curve, but the sign of each double tangent can change if we choose a different $H^{\prime}$. If $k_{1}^{\prime}$ denotes the number of positive double tangents and $k_{2}^{\prime}$ the number of negative double tangents, then we have the following formula.

$$
k_{1}^{\prime}-k_{2}^{\prime}=|v|-1
$$

Moreover, Bjerre also proves that the number of signed double points and signed double tangents is further related for this family, because it holds that:

$$
k_{1}^{\prime}=k_{1} \quad k_{2}^{\prime}=k_{2}
$$

It follows from these results that a curve $\gamma$ in $\mathfrak{B}$ with index $v$ that has the minimum number of $v-1$ double points, which must all be positive, has also the minimum number of $v-1$ double tangents, which must all be positive as well. In addition, if one increases by $q$ the number of positive double points of $\gamma$, by continuously moving its arcs for example, then it must also get $q$ extra negative double points, $q$ extra positive double tangents and $q$ extra negative double tangents. So basically these four objects come together in a bundle.
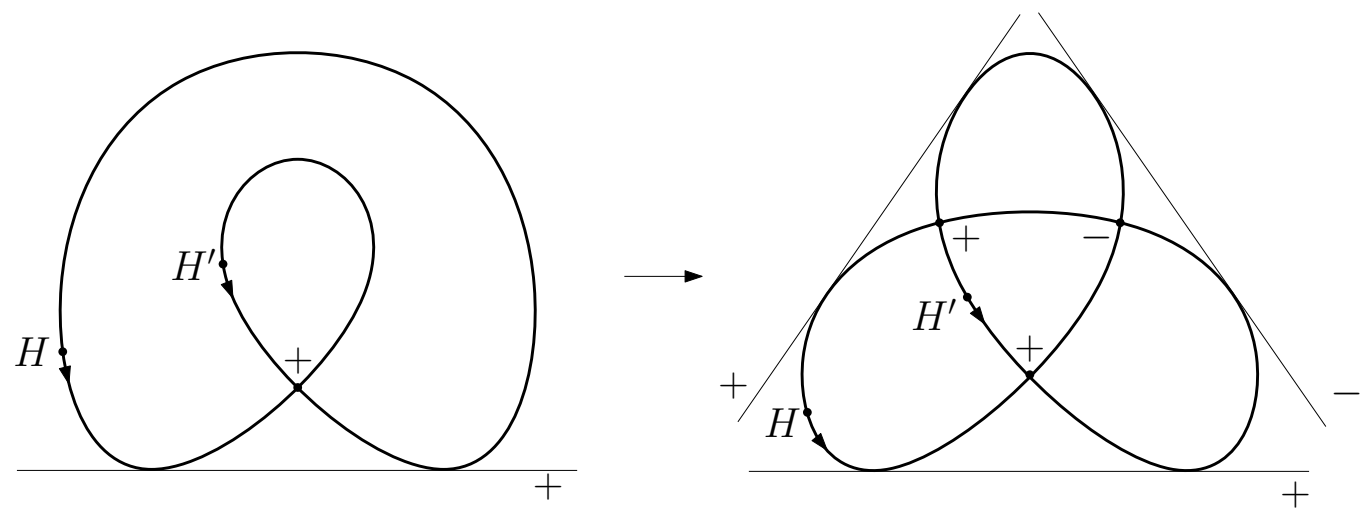
Bjerre comments on the subset $S \subset \mathfrak{B}$ of curves with the minimum number of double points. As an outcome of his arguments to prove the equation $k_{1}-k_{2}=|v|-1$, we get that a curve $\gamma \in S$ must be of a very specific shape. After setting $H \in h \cap \gamma$ and $H^{\prime} \in h^{\prime}$, we can consider that $\gamma$ is divided into two arcs, namely $H H^{\prime}$ and $H^{\prime} H$. The finding is that both of them must be simple spirals and since there can only be positive double points, one only gets closer to the kernel going along $H^{\prime}$, whereas the opposite holds for $H^{\prime} H$. Consequently, there isn't much room for variations. Here are a couple of examples.
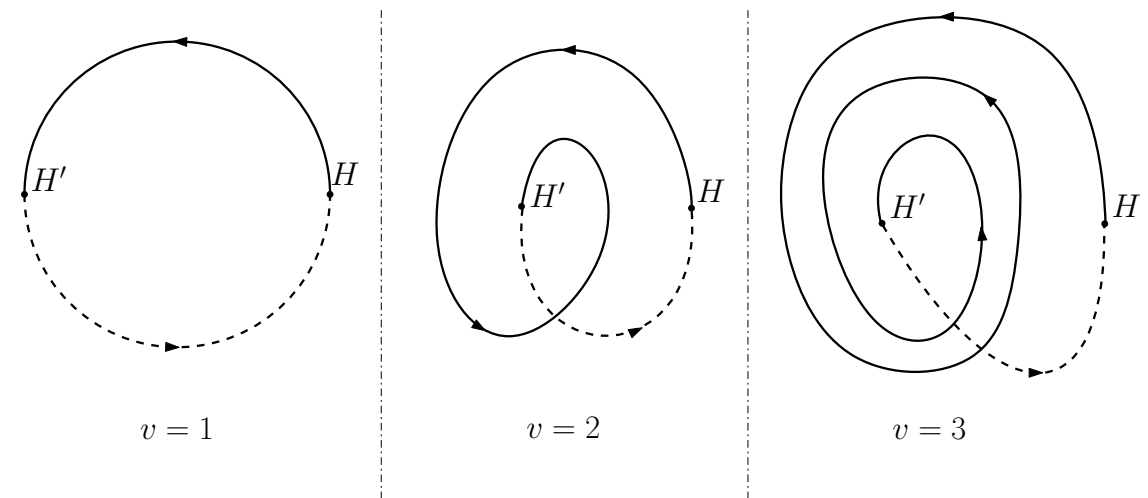

\section{3}

\section{Possible routes for generalisation}

There are a couple ways to develop this theory presented by Bjerre. He published a more general formula for $t-s=d+i$ in another paper (12) in which he considered closed planar curves with cusps.

In a nutshell, the curve $\gamma$ can have two types of cusps. Consider the line $l$ that is the limit of the tangents to $\gamma$ as we approach the cusp $c$. As one passes it, the curve can go to the other side of $l$, in which case we call $c$ a cusp of the first kind, or it can stay on the same half-plane as before, then we call $c$ a cusp of the second kind. Notice that in the latter case the sense of rotation of $\gamma$ is inverted.

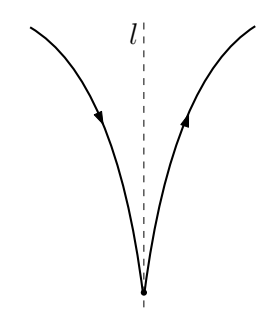

Cusp of the first kind

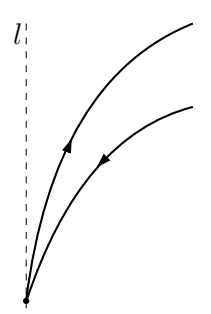

Cusp of the second kind 
If $\gamma$ has $t$ exterior double tangents, $s$ interior double tangents, $d$ double points, $i$ inflectional points, $c_{1}$ cusps of the first kind and $c_{2}$ cusps of the second kind, then

$$
t-s=d+\frac{1}{2} i+c_{1}+\frac{1}{2} c_{2}
$$

Another path we could take to expand the investigation is to consider curves on the projective plane. In fact, when Bjerre originally presented the family $\mathfrak{B}$, he defined it as a class of closed curves in the projective plane. However, the property that there must be a line $u$ such that the curve $\gamma$ doesn't cross it allows us to set it as the line at infinity and hence we can picture $\gamma$ as a planar curve. In the next and final chapter we will shift our perspective and consider closed curves on the projective plane. 


\section{7}

\section{Closed curves on the projective plane}

\section{1}

\section{Relocating to the projective plane}

The aim of this chapter is to introduce to the projective plane the study done so far on generic closed curves. We shall apply the theory developed in the previous chapters and analyse a couple of examples in the hope that new ideas might arise. We'll have to adapt many definitions to this new setting, starting by the curves themselves.

Our current object of study are immersions $i: \mathbb{S}^{1} \rightarrow \mathbb{R} \mathbb{P}^{2}$ into the real projective plane. As before, we are interested in generic curves, so they can only have double points and double tangents as singularities. Notice that if there exists a line $u$ in $\mathbb{R}^{2}$ that does not intersect a curve $\gamma$, then one can apply a projective transformation in order to set it as the line at infinity and thus we essentially get $\gamma$ as a curve in $\mathbb{R}^{2}$. We are already familiar with this type of curve, so let's consider one that cannot be drawn in the plane.

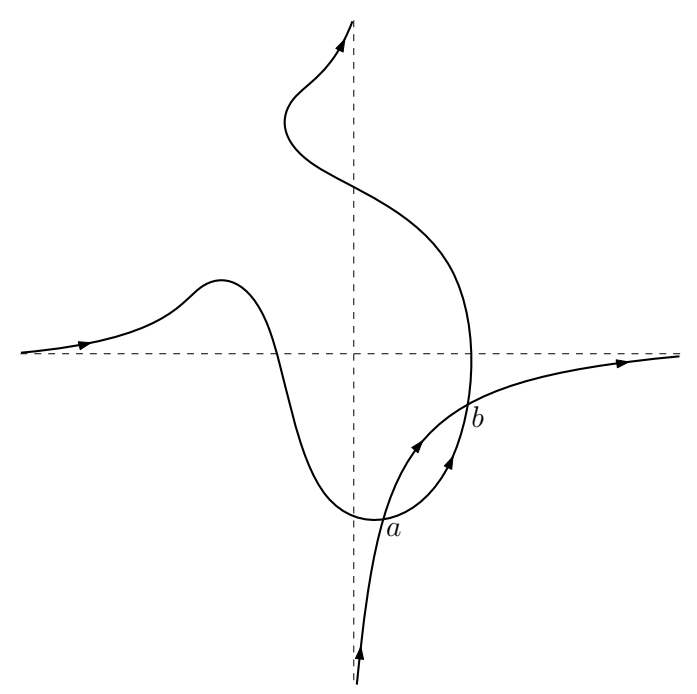

We can define the cyclic word of double points for projective curves too. Notice that the word for the curve above is $a b a b$, which is an impossible word for planar curves since it goes against Gauss's necessary condition presented on chapter 2. Thus we can already conclude that there are less restrictions to the words of projective curves. 
Since the projective plane is a non-orientable surface, we will not give signs to the double points, nor consider signed cyclic words. It would be interesting to adapt the problem set in chapter 1 for this new space and seek necessary conditions on the combinatorics of a cyclic word for it to be realizable by some curve on the projective plane. Similar topological arguments might provide some results, but this is not the goal of this work.

If we wish to talk about locally convex curves, we must understand how the concavity behaves when we pass through infinity and how to identify inflectional points at infinity. In order to do so, it is worthwhile to consider the universal covering space of $\mathbb{R P}^{2}$ which is the sphere $\mathbb{S}^{2}$. It is a double cover that sends antipodal points on the sphere to the same point on the projective plane. Therefore, we can picture the equator sent to the line at infinity and each hemisphere as a copy of the remainder of the projective plane. Now if we draw a small convex arc that crosses the equator, we can see how its antipodal pair reenters the original hemisphere and thus understand what a locally convex curve looks like on the projective plane.

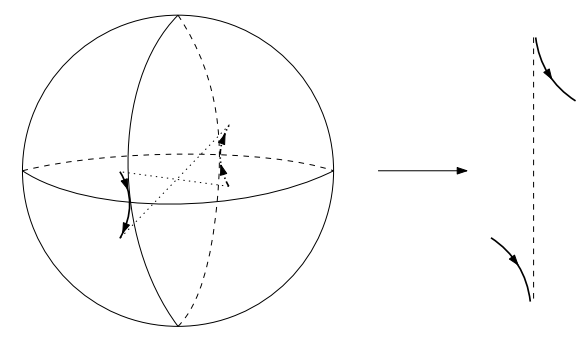

Let's study an example of a locally convex curve $\gamma$ to explore some of its properties and to get used to working with curves on the projective plane. Its cyclic word is abcdeafecdbf, which again defies Gauss's necessary condition because there is an odd number of letters between the two copies of $c$.

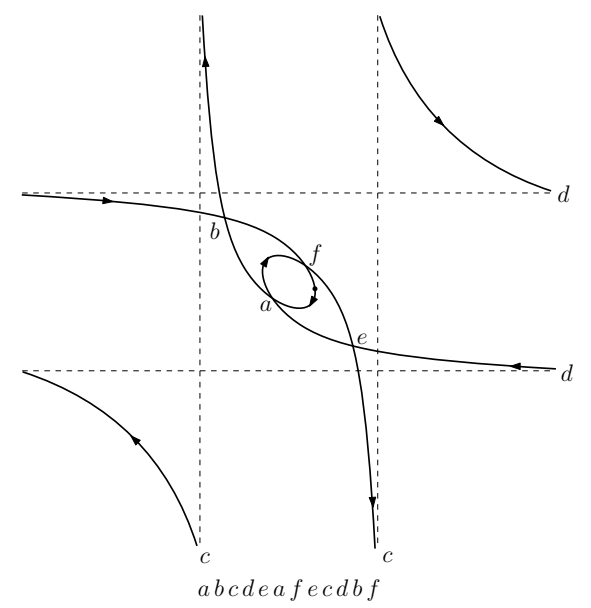


There are some points at infinity that we cannot draw. Actually, the point at infinity by which the curve passes can be more easily spotted if the curve is asymptotic to a line, so we will only consider such a kind of curve. It is important to pay attention to the direction of the asymptotes, since it determines the point at infinity. Thus, in our example there are in fact two double points at infinity $(c$ and $d)$, since there are two vertical asymptotes and two horizontal ones.

This is a good opportunity to describe the tangent lines to the curve at a point at infinity. Consider the double point $c$. It is reached by vertical asymptotes. If one tracks the tangents to $\gamma$ as we go to $c$, there is a clear limit line, its very own asymptote. That line passes over the point $c$ and it is tangent to $\gamma$ at that crossing. Each vertical line represents a different possible tangent at $c$, and there is also a last possible tangent which is the line at infinity. For example, if we consider the parabola $y=x^{2}$ as a curve on the projective plane, it also passes by that same point $c$ but the curve is tangent to the line at infinity. One can perceive this by looking at the tangent lines to the parabola as one moves towards infinity, their direction converges to the vertical but no vertical line on the euclidean plane is the limit of those tangents, the limit "slips" and "hides" at infinity.

Going back to $\gamma$, we now see that it passes by $c$ twice and that each crossing has a distinct tangent, so the curve has no self-tangency, as it should be for any generic curve. It can be useful to leave some double points at infinity, but there are occasions in which it is preferable to have them all in plain sight. Naturally, one just has to move $\gamma$ slightly to push every singularity out of infinity. It is a good exercise to picture that in our example.

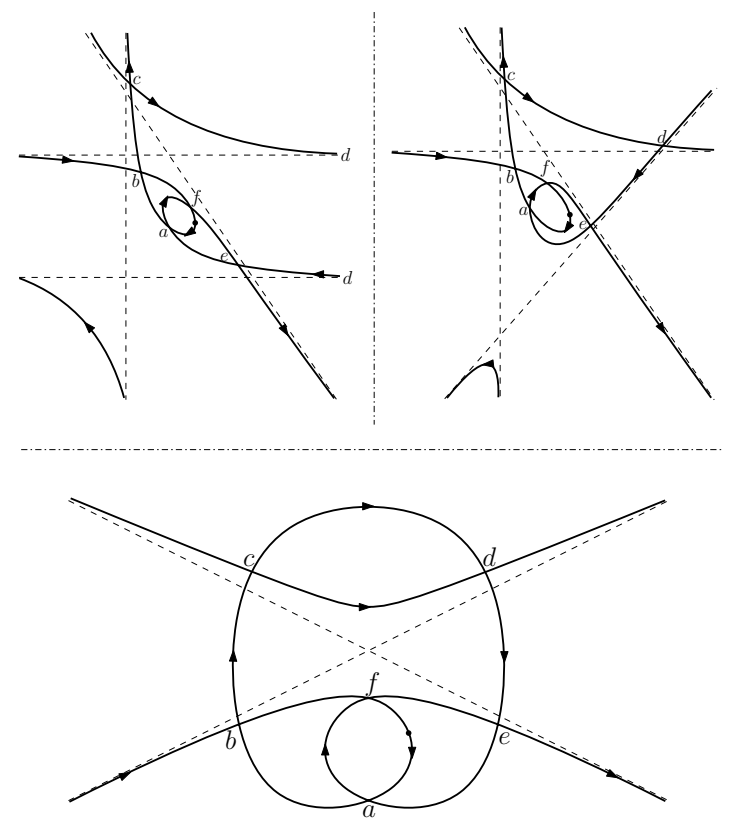


As illustrated above, if one gently budges one of the asymptotes, the double point appears on the plane. First we did it for $c$, then $d$ and finally we pulled the small arc on the bottom through infinity to get a prettier picture of $\gamma$. Notice that by doing so, two asymptotes could be discarded and the figure has become simpler and easier to understand.

Hopefully this example was useful to realize the nature of locally convex curves on the projective plane. A result that can be pointed out is that any locally convex curve must cross the line at infinity an even number of times (tangency to the line at infinity should be counted doubly). This is easy to see why, every time the curve passes through infinity its concavity seen as a planar curve is inverted. Since the curve is closed and it has no inflectional points, the concavity must flip an even number of times.

\section{2}

\section{The word of double tangents}

In the spirit of Bjerre, we will now consider the cyclic words of the tangents of a curve in the projective plane. Each double tangent is designated by a capital letter and as one traverses the curve, its tangent crosses each double tangent twice. By writing down the letters along the way, one gets a cyclic word for the double tangents of the curve. Let's inspect $\gamma$ from the last example and uncover its word of tangents.

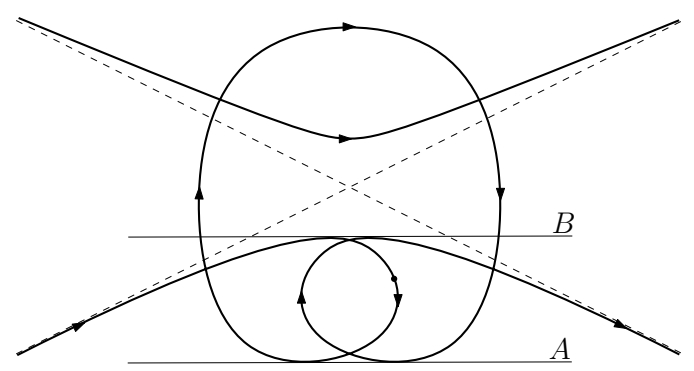

It turns out it has quite a simple word for its double tangents $A A B B$. As we did in chapter 3 , it would be nice to gather curves with the same words into equivalence classes. This time each curve has two words, one for its double points and another one for its double tangents, so the classes will be more specific.

Previously, two curves were in the same class if one could connect them with a path of immersions that avoided all singular events. In that case, we were not paying much attention to the tangents of the curves, so we only considered the singular events described by Arnold, namely triple crossings of the immersion and self-tangencies. This time, however, we must also prevent the crossing of triple tangents. 
Definition 7.1 Two closed generic oriented immersed curves on the projective plane are in the same equivalence class if and only if there is a connecting path of immersions that avoids all singular events between them, including triple tangent crossings.

In chapter 3 we've proved a one-to-one correspondence between cyclic words of realizable curves on the plane and the classes defined then. In the present case, it is not clear whether there is also a one-to-one correspondence, although each class is uniquely represented by an ordered pair of cyclic words since every singular event is prohibited.

In the following figure there are two curves that we used to consider as the same, but that have distinct words for their double tangents due to a triple tangent crossing. From now on, these curves are in distinct classes, so they are no longer the same.

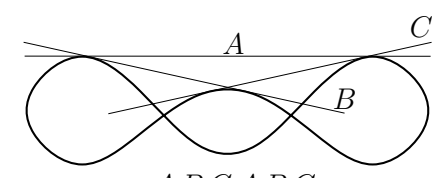

$A B C A B C$

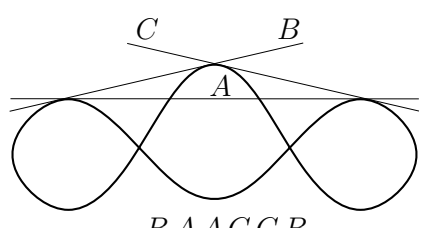

$B A A C B$

\section{3}

\section{The dual curve}

There is a very interesting and useful notion of duality on $\mathbb{R P}^{2}$ that interchanges points and lines. This map is a polarity, which means that it is a duality and also an involution. If we consider homogeneous coordinates $(x: y: z)$ for the projective plane, then the point $(a: b: c)$ is mapped to the line of equation $a x+b y+c z=0$, and vice versa. Geometrically, this correlation is directly related to the concept of reciprocation, that takes points to their polars and lines to their poles. For more information on that, see (13).

If we consider a generic closed curve $\gamma$ on the projective plane, it is possible henceforth to define its dual curve $\gamma^{*}$. To do so, apply the polarity to the envelope of tangent lines to $\gamma$, the result is a new closed curve on the projective plane.

Interestingly, there is a connection between certain elements of $\gamma$ and $\gamma^{*}$. Firstly, their double points and double tangents are interchanged, i.e., each double point of $\gamma$ becomes a double tangent of $\gamma^{*}$ and each double tangent of $\gamma$ turns into a double point of $\gamma^{*}$. Furthermore, the same phenomenon happens between inflectional points and cusps. Therefore, if we wish to avoid cusps, we should focus on locally convex curves. Finally, if we consider curves in the 
family $\mathfrak{B}$ defined by Bjerre and presented in chapter 5 , the exterior domain $\omega$ and the kernel $\omega^{\prime}$ are also swapped. Consequently, one can already realise that if $\gamma \in \mathfrak{B}$, then $\gamma^{*} \in \mathfrak{B}$.

It is a fun exercise to pick a curve $\gamma$ and try to draw $\gamma^{*}$. It is a great strategy to first write down both cyclic words derived from it, they will serve as guidelines. It is also a good ideia to look for an exterior domain and a kernel. If $\gamma$ has no kernel, then we already know that $\gamma^{*}$ has no exterior domain, and hence it cannot be drawn as a planar curve, passing through infinity is inevitable. Let's observe some examples.

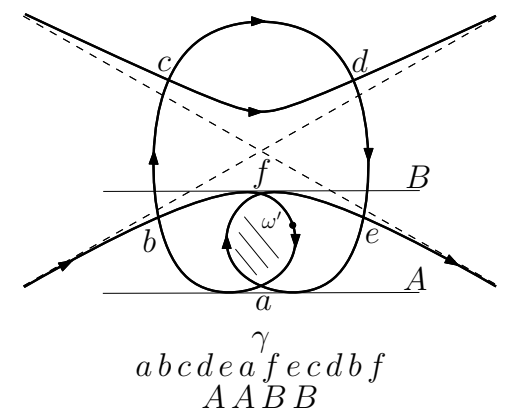

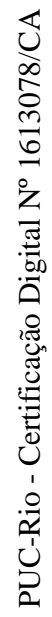
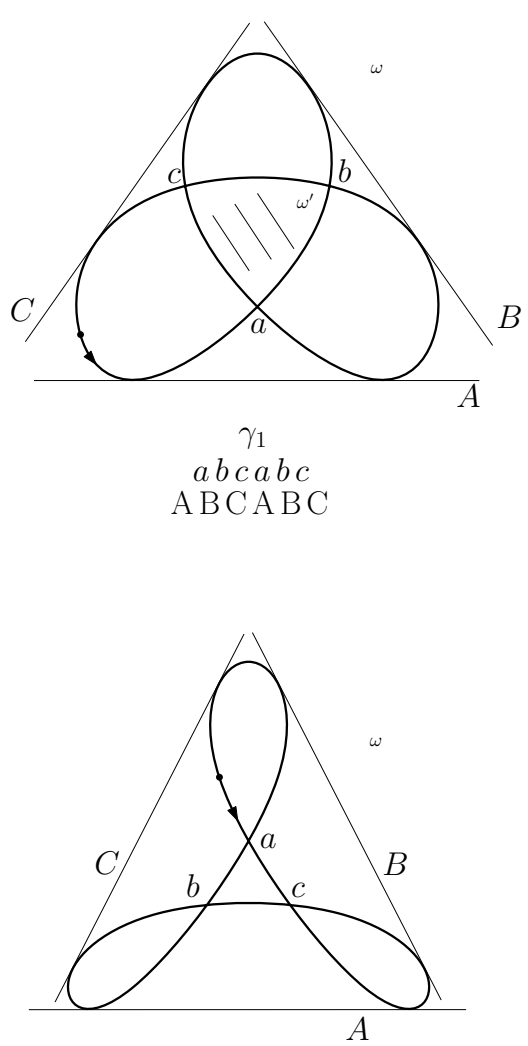

$\gamma_{2}$ $\mathrm{ABCABC}$

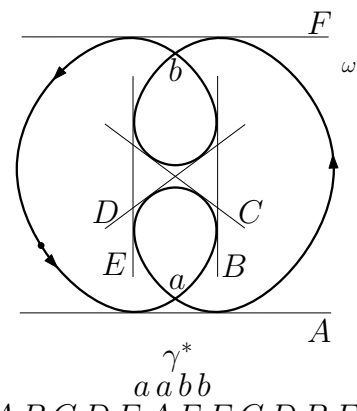

$A B C D E A F E C D B F$
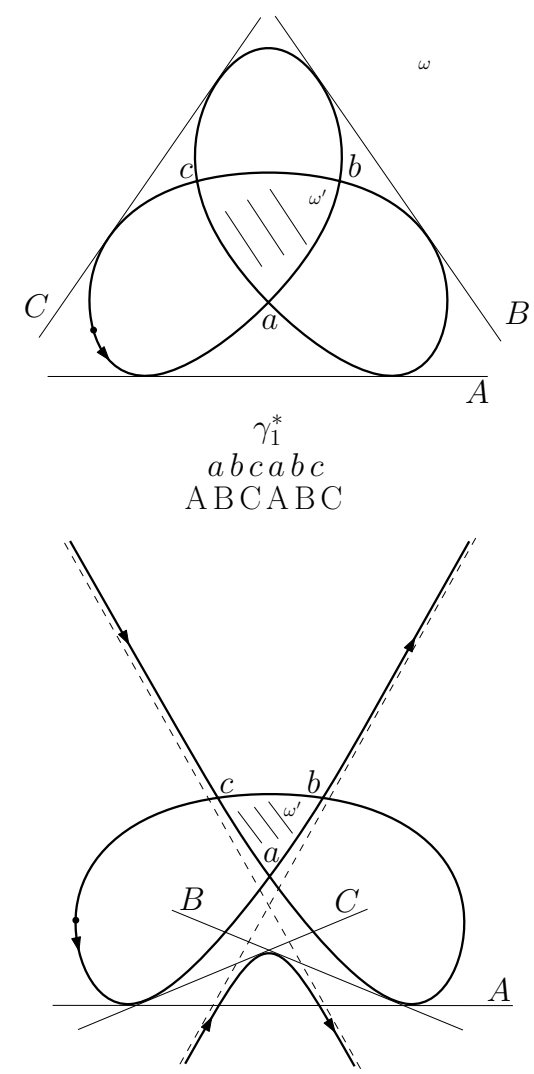

$\gamma_{2}^{*}$ $a b c a b c$ $\mathrm{ACCBBA}$ 
Compare the last two pairs of dual curves. The difference between $\gamma_{1}$ and $\gamma_{2}$ is a triple point crossing. Notice that the corresponding difference between $\gamma_{1}^{*}$ and $\gamma_{2}^{*}$ is a triple tangent crossing. In addition, as from $\gamma_{1}$ to $\gamma_{2}$ the kernel is undone, from $\gamma_{1}^{*}$ to $\gamma_{2}^{*}$ the exterior domain is lost.

There is a vast interest in self-dual curves, that is, curves that are projectively equivalent to their duals. Arnold himself has manifested his curiosity as he has posed the following problem among his extensive collection of open problems: Problem 1994-17. Find all projective curves equivalent to their duals. The answer seems to be unknown even in $\mathbb{R P}^{2}(14)$.

We will adapt this question to the classes of projective curves that we have defined. The duality sends a class represented by the words $\left(w_{1}, w_{2}\right)$ to another class whose words are $\left(w_{2}, w_{1}\right)$. So we will call self-dual class those classes that remain invariant under duality. One can easily see that a necessary condition is that $w_{1}=w_{2}$.

So far, we have seen an example of a self-dual class whose words were both $a b c a b c$. Such a curve was in the family $\mathfrak{B}$ and it seems that the duality restricted to $\mathfrak{B}$ is the identity. However, we know that there are self-dual curves which are locally convex but are not in $\mathfrak{B}$. We thank Prof. Nicolau Saldanha for the following example.

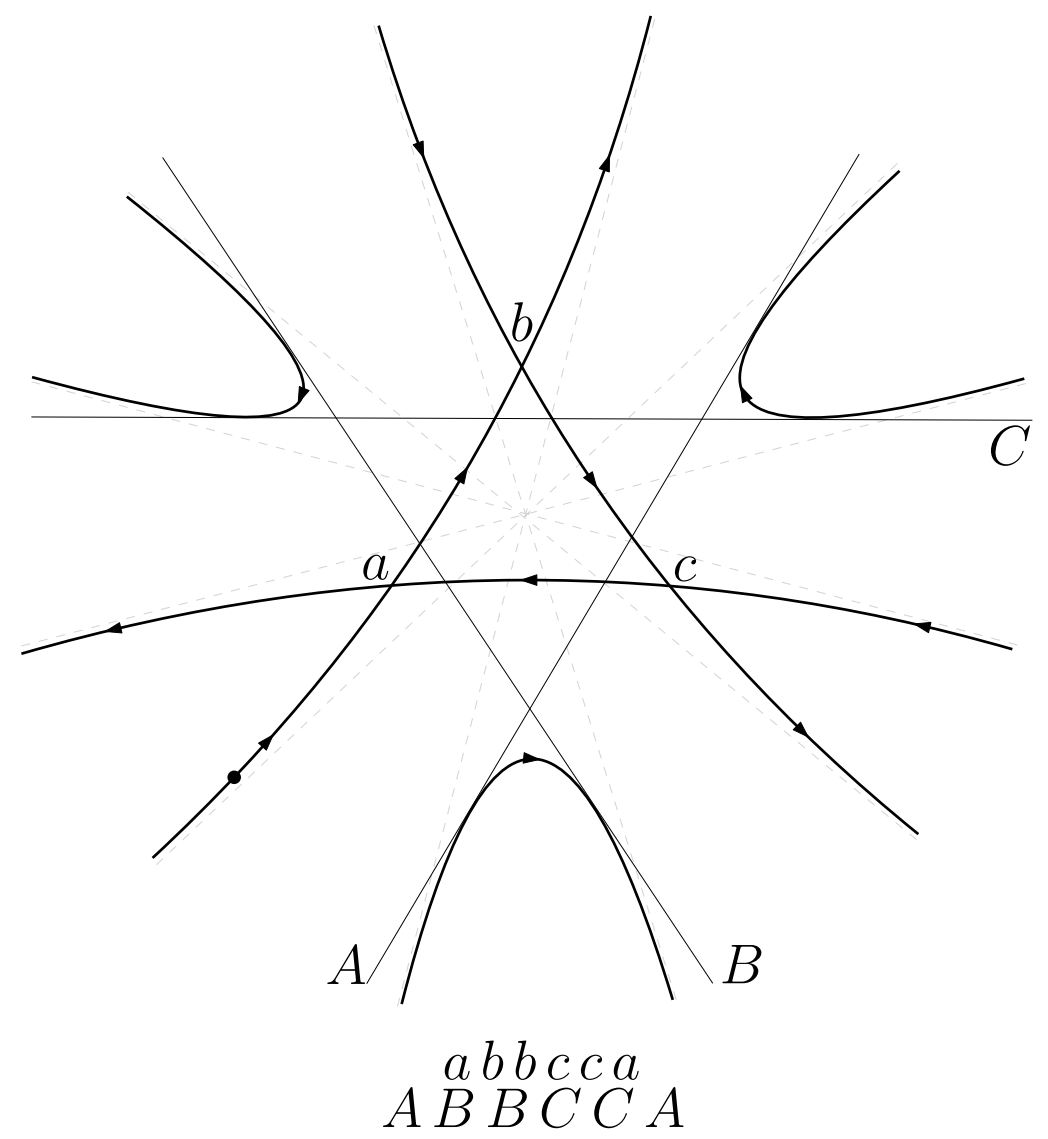


Due to Bjerre's formula $t-s=d+\frac{1}{2} i+c_{1}+\frac{1}{2} c_{2}$, one can see that if $\gamma$ is a planar curve and it has any inflectional point or cusp, then it must have more double tangents than double points, thus it cannot be a self-dual curve. Nevertheless, this formula was deduced for planar curves, so it does not prevent a curve with cusps and inflections but no exterior domain from being self-dual.

Let's consider an algebraic example, the curve $\gamma$ on $\mathbb{R P}^{2}$ defined by the equation $z y^{2}=x^{3}$. The planar view for $z=1$ has a cusp on $(x=0, y=0)$, since the equation reduces to $y^{2}=x^{3}$. One can already check that this curve has no exterior domain, even vertical lines $x=c, c<0$ intersect $\gamma$ at infinity. To see where the curve passes at infinity, one just has to take $z=0$, which yields $x^{3}=0$, thus the only point at infinity is $(0: 1: 0)$.

Now changing our perspective to the plane $y=1$, we'll have the said point $(0: 1: 0)$ right at the center of the xz-plane, thus we can analyse its properties. The new equation is $z=x^{3}$, so we can already draw some pictures and reach some conclusions.

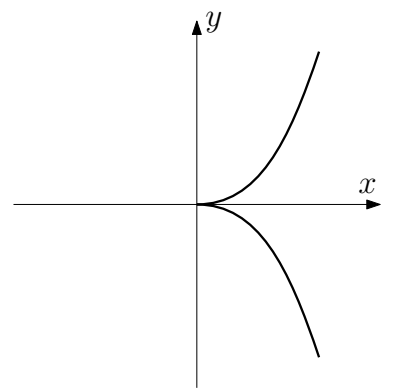

$z=1$

$y^{2}=x^{3}$

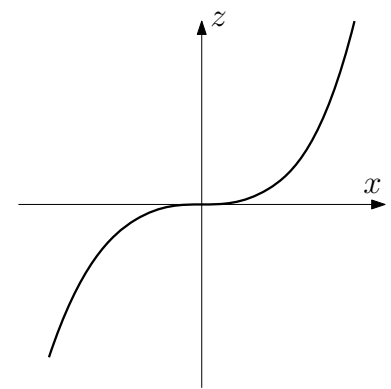

$y=1$

$z=x^{3}$

The curve has an inflectional point at $(0: 1: 0)$ and a cusp at $(0: 0: 1)$, those are all of its singularities, $\gamma$ has no double points or double tangents. Therefore this is an exemple of a self-dual curve that is not locally convex.

We have only scratched the surface of this subject. To further develop the study some authors considered the more restrictive problem of self-dual algebraic curves; there is some sophisticated mathematics on the matter. 


\section{Bibliography}

[1] GHYS, E.. A singular mathematical promenade. ENS Éditions, Lyon, 2017.

[2] ARNOLD, V. I.. Topological invariants of plane curves and caustics, volumen 5 de University Lecture Series. American Mathematical Society, Providence, RI, 1994. Dean Jacqueline B. Lewis Memorial Lectures presented at Rutgers University, New Brunswick, New Jersey.

[3] DO CARMO, M. P.. Differential geometry of curves and surfaces. Prentice-Hall, Inc., Englewood Cliffs, N.J., 1976. Translated from the Portuguese.

[4] THOMASSEN, C.. The jordan-schonflies theorem and the classification of surface. The American Mathematical Monthly, 99(2):116-131, 1992.

[5] BENDIXSON, I.. Sur les courbes définies par des équations différentielles. Acta Math., 24(1):1-88, 1901.

[6] WHITNEY, H.. On regular closed curves in the plane. Compositio Math., 4:276-284, 1937.

[7] BURMAN, Y.; POLYAK, M.. Geometry of Whitney-type formulas. Mosc. Math. J., 3(3):823-832, 1197, 2003. \{Dedicated to Vladimir Igorevich Arnold on the occasion of his 65th birthday .

[8] BURMAN, Y.; POLYAK, M.. Whitney's formulas for curves on surfaces. Geom. Dedicata, 151:97-106, 2011.

[9] MIKHALKIN, G.; POLYAK, M.. Whitney formula in higher dimensions. J. Differential Geom., 44(3):583-594, 1996.

[10] FABRICIUS-BJERRE, F.. On the double tangents of plane closed curves. Math. Scand, 11:113-116, 1962.

[11] FABRICIUS-BJERRE, F.. On a class of locally convex closed curves. Ann. Mat. Pura Appl. (4), 55:47-54, 1961. 
[12] FABRICIUS-BJERRE, F.. A relation between the numbers of singular points and singular lines of a plane closed curve. Math. Scand., 40(1):20-24, 1977.

[13] GIBSON, C. G.. Elementary geometry of algebraic curves: an undergraduate introduction. Cambridge University Press, Cambridge, 1998.

[14] ARNOLD, V. I.. Arnold's problems. Springer-Verlag, Berlin; PHASIS, Moscow, 2004. Translated and revised edition of the 2000 Russian original, With a preface by V. Philippov, A. Yakivchik and M. Peters. 